\title{
On the new critical exponent for the nonlinear Schrödinger equations
}

\author{
Nakao Hayashi and Pavel I. Naumkin
}

\begin{abstract}
We consider the Cauchy problem for the $p$ th order nonlinear Schrödinger equation in one space dimension

$$
\left\{\begin{array}{c}
i u_{t}+\frac{1}{2} u_{x x}=|u|^{p}, x \in \mathbf{R}, t>0, \\
u(0, x)=u_{0}(x), x \in \mathbf{R},
\end{array}\right.
$$

where $p>p_{s}=\frac{3+\sqrt{17}}{2}$. We reveal that $p=4$ is a new critical exponent with respect to the large time asymptotic behavior of solutions. We prove that if $p_{s}<p<4$, then the large time asymptotics of solutions essentially differs from that for the linear case, whereas it has a quasilinear character for the case of $p>4$.
\end{abstract}

Mathematics Subject Classification (1991). 35B40, 35Q55.

Keywords. Asymptotics of solutions, Nonlinear Schrödinger equation, New critical exponent.

\section{Introduction}

We consider the Cauchy problem for the $p$ th order nonlinear Schrödinger equation in one space dimension

$$
\left\{\begin{array}{l}
i u_{t}+\frac{1}{2} u_{x x}=|u|^{p}, x \in \mathbf{R}, t>0, \\
u(0, x)=u_{0}(x), x \in \mathbf{R}
\end{array}\right.
$$

with $p>p_{s}=\frac{3+\sqrt{17}}{2}$. Our aim in this paper is to prove that $p=4$ is a critical exponent with respect to the large time asymptotic behavior of solutions to the Cauchy problem (1.1). We will prove that if $p_{s}<p<4$, then the large time asymptotics of solutions essentially differs from that for the linear case, whereas it has a quasilinear character for the case of $p>4$. In the general space dimension $n \geq 1$ we conjecture that, for the nonlinearity of the form $|u|^{p}$, the critical exponent is $2+\frac{2}{n}$. This is an interesting new phenomena, comparing with other types of nonlinearities, for example standard gauge invariant nonlinearity $|u|^{p-1} u$, where the critical exponent in the sense of the large time 
asymptotics of solutions is $1+\frac{2}{n}$ (see, e.g. [7,11]). This phenomena is caused by the special resonant property of the nonlinearity $|u|^{p}$, which leads to a singularity $|\xi|^{-\frac{n}{2}\left(2+\frac{2}{n}-p\right)}$ at the origin for the solution in the impulse representation (see Eqs. 1.3 and 1.4 below). The difficulty in the study of the large time asymptotic behavior of solutions to the Cauchy problem (1.1) is that the nonlinearity $|u|^{p}$ is not gauge invariant, namely, it does not satisfy the property $\mathcal{N}\left(u e^{i \theta}\right)=e^{i \theta} \mathcal{N}(u)$ for all $\theta \in \mathbf{R}$. This fact does not allow us to apply directly the operator $\mathcal{J}=x+i t \partial_{x}$, which plays a crucial role in the large time asymptotic behavior of solutions.

Global existence of small solutions to nonlinear Schrödinger equations was studied extensively via the energy estimates and dispersion properties of the Schrödinger evolution group (see, e.g. $[16,17]$ ). In papers $[15,16]$, the global in time existence of small solutions to nonlinear Schrödinger equations was proved in general space dimension $n$ in the case of the power nonlinearity $|u|^{p}$ with $p>p_{s}(n)=\frac{n+2+\sqrt{n^{2}+12 n+4}}{2 n}$. The sharp time decay estimate $\|u(t)\|_{\mathbf{L}^{p+1}} \leq C|t|^{-\frac{n}{2}\left(\frac{p-1}{p+1}\right)}$ for small solutions to the nonlinear Schrödinger equations was also obtained in [16] since the method is based on the $\mathbf{L}^{p+1}-\mathbf{L}^{\frac{p+1}{p}}$-time decay estimate for the free Schrödinger evolution group. These methods are not sufficient for studying the sharp $\mathbf{L}^{\infty}$-time decay properties of solutions. To reveal it, a special oscillating structure of the nonlinearity should be taken into account. We note that exponent $p_{s}(n)$ was discovered in paper [6], concerning the nonlinear wave equation with nonlinearity $|u|^{p}$, where the global existence of small solutions was proved for the case of $p>p_{s}(n-1)$ and the blow up of solutions was shown for $p<p_{s}(n-1)$, when $n=3$. This result was generalized to the space dimensions $n \geq 2$ in papers [3-5,13]. The blow up result was established for the critical case $p=p_{s}(n-1)$ in $[12,18]$. Thus $p_{s}(n-1)$ is the critical exponent with respect to the global existence of small solutions for the nonlinear wave equation (the shift of the dimension is caused by the difference in the time decay rate of the free Schrödinger and wave evolution groups). We do not know if $p_{s}(n)$ is indeed critical with respect to the global existence for the case of the nonlinear Schrödinger equation with a power like nonlinearity of the form $|u|^{p}$. The blow up result was proved in [10] in the case of $1<p \leq 1+\frac{2}{n}$ by using the test function method from [19]. As far as we know there is no any global result for $1+\frac{2}{n}<p \leq p_{s}(n)$.

To state our result precisely we give some notations. We denote the usual Lebesgue space by $\mathbf{L}^{p}$ with the norm $\|\phi\|_{\mathbf{L}^{p}}=\left(\int_{\mathbf{R}}|\phi(x)|^{p} d x\right)^{1 / p}$ if $1 \leq p<\infty$ and $\|\phi\|_{\mathbf{L}^{\infty}}=\operatorname{ess.sup}\{|\phi(x)| ; x \in \mathbf{R}\}$. The weighted Sobolev space is defined by $\mathbf{H}_{p}^{m, k}=\left\{\phi \in \mathbf{L}^{2}:\left\|\langle x\rangle^{k}\left\langle i \partial_{x}\right\rangle^{m} \phi\right\|_{\mathbf{L}^{p}}<\infty\right\}$, where $m, k \in \mathbf{R}^{+}, 1 \leq p \leq$ $\infty,\langle x\rangle=\sqrt{1+|x|^{2}}$. For simplicity we write $\mathbf{H}^{m, s}=\mathbf{H}_{2}^{m, s}, \mathbf{H}^{m}=\mathbf{H}_{2}^{m, 0}$. Let $\mathbf{C}(\mathbf{I} ; \mathbf{B})$ be the space of continuous functions from a time interval $\mathbf{I}$ to a Banach space B. Different positive constants might be denoted by the same letter $C$.

In the first theorem we state global existence of solutions and existence of the usual scattering states in the case of $p>4$. 
Theorem 1.1. Let $p>4$. Let the initial data $u_{0} \in \mathbf{H}^{\nu} \cap \mathbf{H}^{0, \nu}, \frac{1}{2}<\nu<1$ and $\left\|u_{0}\right\|_{\mathbf{H}^{\nu} \cap \mathbf{H}^{0, \nu}}=\varepsilon$ be small. Then there exists a unique global solution $u \in \mathbf{C}\left(\mathbf{R}^{+}, \mathbf{H}^{\nu} \cap \mathbf{H}^{0, \nu}\right)$ to the Cauchy problem (1.1) which satisfies the time decay estimate $\|u\|_{\mathbf{L}^{q}} \leq C \varepsilon t^{-\frac{1}{2}+\frac{1}{q}}$ for $2 \leq q \leq \infty$. Moreover there exists a unique final state $u_{+} \in \mathbf{H}^{0, \nu}$ with $\frac{1}{2}<\nu<1$, such that

$$
\left\|u(t)-e^{\frac{i x^{2}}{2 t}} \frac{1}{\sqrt{i t}} \widehat{u_{+}}\left(\frac{x}{t}\right)\right\|_{\mathbf{L}^{q}} \leq C \varepsilon t^{-\frac{1}{2}\left(1-\frac{2}{q}\right)-\gamma}
$$

for $2 \leq q \leq \infty$ with some small $\gamma>0$.

Remark 1.1. Note that $e^{\frac{i x^{2}}{2 t}} \frac{1}{\sqrt{i t}} \widehat{u_{+}}\left(\frac{x}{t}\right)$ is the main term of the large time asymptotics of solutions to the linear problem. Thus the large time asymptotics of solutions to (1.1) has a quasilinear character for the case of $p>4$.

Next we consider the case of $p_{s}<p<4$.

Theorem 1.2. Let $p_{s}<p<4$. Let the initial data $u_{0} \in \mathbf{H}^{3,2}$ and $\left\|u_{0}\right\|_{\mathbf{H}^{3,2}}=\varepsilon$ be small. Then there exists a unique global solution $u \in \mathbf{C}\left(\mathbf{R}^{+}, \mathbf{H}^{3,2}\right)$ to the Cauchy problem (1.1) which satisfies the estimate

$$
\|u(t)\|_{\mathbf{L}^{q}} \leq C \varepsilon\left\{\begin{array}{l}
t^{-\frac{1}{2}+\frac{1}{q}} \quad \text { for } 2 \leq q<\frac{2}{4-p}, \\
t^{\frac{1}{2}-\frac{p}{4}+\frac{1}{2 q}} \quad \text { for } \frac{2}{4-p}<q \leq \infty .
\end{array}\right.
$$

Moreover there exists a unique final state $\widehat{u_{+}} \in \mathbf{L}^{2} \cap \mathbf{L}^{\frac{2}{4-p}-\gamma}$, with some small $\gamma>0$, such that

$$
\left\|u(t)-e^{\frac{i x^{2}}{2 t}} \frac{1}{\sqrt{i t}} \widehat{u_{+}}\left(\frac{x}{t}\right)\right\|_{\mathbf{L}^{q}} \leq C \varepsilon t^{-\frac{1}{2}\left(1-\frac{2}{q}\right)-\gamma}
$$

for $2 \leq q \leq \frac{2}{4-p}-\gamma$. Also there exists a unique function $\Psi_{+} \in \mathbf{L}^{\infty} \cap \mathbf{L}^{\frac{2}{4-p}+\gamma}$, such that the asymptotics

$$
\left\|u(t)-e^{\frac{i x^{2}}{2 t}} \frac{1}{\sqrt{i t}} t^{1-\frac{p}{4}} \Psi_{+}\left(\frac{x}{\sqrt{t}}\right)\right\|_{\mathbf{L}^{q}} \leq \varepsilon t^{\frac{1}{2}-\frac{p}{4}+\frac{1}{2 q}-\gamma}
$$

is true as $t \rightarrow \infty$, for $\frac{2}{4-p}+\gamma \leq q \leq \infty$.

Remark 1.2. Note that the time decay rate for the $\mathbf{L}^{q}$-norm with $2 \leq q<\frac{2}{4-p}$, is the same as that for the linear case, whereas it is slower in the case of $\frac{2}{4-p}<q \leq \infty$. The $\mathbf{L}^{\frac{2}{4-p}}$-time decay estimate of solutions can also be obtained if we introduce a logarithmic correction. However the sharp $\mathbf{L}^{\frac{2}{4-p}}$-time decay is not known. The large time asymptotics of solutions in $\mathbf{L}^{q}$ with $\frac{2}{4-p}<q \leq \infty$ essentially differs from that for the linear case. Moreover it shows that the time decay rates of the $\mathbf{L}^{q}$-norms are optimal. We also note that

$$
\left\|\frac{1}{\sqrt{i t}} t^{\frac{4-p}{4}} \Psi_{+}\left(\frac{x}{\sqrt{t}}\right)\right\|_{\mathbf{L}^{q}}=t^{\frac{1}{2}-\frac{p}{4}+\frac{1}{2 q}}\left\|\Psi_{+}\right\|_{\mathbf{L}^{q}}
$$

and 


$$
\left\|\frac{1}{\sqrt{i t}} \widehat{u_{+}}\left(\frac{x}{t}\right)\right\|_{\mathbf{L}^{q}}=t^{-\frac{1}{2}\left(1-\frac{2}{q}\right)}\left\|\widehat{u_{+}}\right\|_{\mathbf{L}^{q}},
$$

where the decay rates coincide $\frac{1}{2}-\frac{p}{4}+\frac{1}{2 q}=-\frac{1}{2}\left(1-\frac{2}{q}\right)$, when $q=\frac{2}{4-p}$.

Remark 1.3. The critical exponent $p=4$ could be also considered by our method. We expect that the time decay rate of the $\mathbf{L}^{\infty}$-norm of solutions has a logarithmic correction comparing with the linear case.

We now briefly describe our strategy. As in our previous papers, we use the factorization technique from [9] for the free Schrödinger evolution group

$$
\mathcal{U}(t)=e^{\frac{i t}{2} \Delta}=M(t) \mathcal{D}(t) \mathcal{V}(t) \mathcal{F},
$$

where the multiplication factor $M(t)=e^{\frac{i|x|^{2}}{2 t}}$, the dilation operator $(\mathcal{D}(t) \phi)(x)$ $=(i t)^{-\frac{n}{2}} \phi\left(\frac{x}{t}\right)$, and the operator $\mathcal{V}(t)=\mathcal{F} M(t) \mathcal{F}^{-1}$. Here $\mathcal{F} \phi=$ $\widehat{\phi}=(2 \pi)^{-\frac{n}{2}} \int_{\mathbf{R}^{n}} e^{-i(x \cdot \xi)} \phi(x) d x$ denotes the Fourier transform of the function $\phi$, and $\mathcal{F}^{-1}$ is the inverse Fourier transformation defined by $\mathcal{F}^{-1} \phi=$ $(2 \pi)^{-\frac{n}{2}} \int_{\mathbf{R}^{n}} e^{i(x \cdot \xi)} \phi(\xi) d \xi$. Formula (1.2) is very useful for studying the large time asymptotic behavior of solutions to the Schrödinger equations. We have

$$
\mathcal{F U}(-t)=\mathcal{V}(-t) \mathcal{D}^{-1}(t) \bar{M}(t)=i^{n} \mathcal{V}(-t) \bar{E}(t) \mathcal{D}\left(\frac{1}{t}\right)
$$

where $E(t, \xi)=e^{\frac{i t}{2}|\xi|^{2}}$. Since $\mathcal{F} M(t)=(i t)^{\frac{n}{2}} \bar{E}(t)$, we find that

$$
\mathcal{V}(t) \varphi(t)=\left(\frac{i t}{2 \pi}\right)^{\frac{n}{2}} \int_{\mathbf{R}^{n}} e^{-\frac{i t}{2}|\xi-\eta|^{2}} \varphi(t, \eta) d \eta .
$$

We next multiply both sides of $(1.1)$ by $\mathcal{F U}(-t)$ and use the factorization technique

$$
\begin{aligned}
i \varphi_{t} & =\mathcal{F U}(-t)|u|^{p}=i^{n} \mathcal{V}(-t) \bar{E}(t) \mathcal{D}\left(\frac{1}{t}\right)|u|^{p} \\
& =i^{\frac{n}{2}} \mathcal{V}(-t) \bar{E}(t) t^{\frac{n}{2}}|u(t, t x)|^{p}=i^{\frac{n}{2}} \mathcal{V}(-t) \bar{E}(t) t^{\frac{n}{2}(1-p)}\left|\mathcal{D}\left(\frac{1}{t}\right) u\right|^{p} \\
& =i^{\frac{n}{2}} \mathcal{V}(-t) \bar{E}(t) t^{\frac{n}{2}(1-p)}|\mathcal{F} M \mathcal{U}(-t) u|^{p} \\
& =\left(\frac{t}{2 \pi}\right)^{\frac{n}{2}} t^{\frac{n}{2}(1-p)} \int_{\mathbf{R}^{n}} e^{\frac{i t}{2}|\xi-\eta|^{2}} e^{-\frac{i t}{2}|\eta|^{2}}|v(t, \eta)|^{p} d \eta \\
& =(2 \pi)^{-\frac{n}{2}} t^{\frac{n}{2}(2-p)} E \int_{\mathbf{R}^{n}} e^{-i t(\xi \cdot \eta)}|v(t, \eta)|^{p} d \eta
\end{aligned}
$$

where the new dependent variables $\varphi(t)=\mathcal{F U}(-t) u(t)$ and $v(t)=$ $i^{-\frac{n}{2}} \mathcal{F} M \mathcal{U}(-t) u(t)=i^{\frac{n}{2}} \bar{E}(t) \mathcal{D}\left(\frac{1}{t}\right) u(t)$. Thus we transform equation (1.1) to the following form

$$
i \varphi_{t}(t, \xi)=(2 \pi)^{-\frac{n}{2}} t^{\frac{n}{2}(2-p)} E \int_{\mathbf{R}^{n}} e^{-i t(\xi \cdot \eta)}|v(t, \eta)|^{p} d \eta .
$$

By the integrability condition of the factor $t^{\frac{n}{2}(2-p)}$ in Eq. (1.3) we deduce that in the case of the general space dimension $n$ the critical exponent is $2+\frac{2}{n}$. 
However it is an open question for $n \geq 2$, since our approach requires some regularity conditions on the nonlinearity $|u|^{p}$. We studied global in time existence of small solutions to the nonlinear Schrödinger equation in four dimensional case with quadratic interaction $|u|^{2}$ in paper [8], where we also proved the existence of the usual scattering states and showed that the large time asymptotics of solutions differs from that for the linear Schrödinger equation. Thus the quadratic interaction in 4-dimensional case lies below the critical exponent in the sense of time decay of solutions.

In finding the large time asymptotics in the subcritical case $p_{s}<p<2+\frac{2}{n}$ our strategy is to prove that

$$
\varphi(t, \xi)=\Phi(\xi)+R(t, \xi)
$$

where $R(t, \xi)$ is a remainder term and

$$
\Phi(\xi)=\varphi(0, \xi)-i(2 \pi)^{-\frac{n}{2}} \int_{0}^{\infty} \tau^{\frac{n}{2}(2-p)} e^{\frac{i \tau}{2}|\xi|^{2}} \int_{\mathbf{R}^{n}} e^{-i t(\xi \cdot \eta)}|v(t, \eta)|^{p} d \eta d \tau,
$$

from which we show that for large $t$

$$
\begin{aligned}
& \varphi(t, \xi) \simeq \varphi(0, \xi)-i(2 \pi)^{-\frac{n}{2}} \int_{0}^{\infty} \tau^{\frac{n}{2}(2-p)} e^{\frac{i \tau}{2}|\xi|^{2}} \int_{\mathbf{R}^{n}} e^{-i \tau \xi \cdot \eta}|\Phi(\eta)|^{p} d \eta d \tau \\
& \simeq \varphi(0, \xi)-i(2 \pi)^{-\frac{n}{2}} \int_{0}^{\infty} \tau^{\frac{n}{2}(2-p)} \int_{\mathbf{R}^{n}} e^{-i \tau \xi \cdot \eta}|\Phi(\eta)|^{p} d \eta d \tau \\
& =\varphi(0, \xi)-i(2 \pi)^{-\frac{n}{2}}|\xi|^{-\frac{n}{2}\left(2+\frac{2}{n}-p\right)} \int_{0}^{\infty} z^{\frac{n}{2}(2-p)} \int_{\mathbf{R}^{n}} e^{-i z\left(\frac{\xi}{|\xi|} \cdot \eta\right)}|\Phi(\eta)|^{p} d \eta d z \\
& =\varphi(0, \xi)-i(2 \pi)^{-\frac{n}{2}}|\xi|^{-\frac{n}{2}\left(2+\frac{2}{n}-p\right)} A\left(\frac{\xi}{|\xi|}\right)
\end{aligned}
$$

with

$$
A\left(\frac{\xi}{|\xi|}\right)=\int_{0}^{\infty} z^{\frac{n}{2}(2-p)} \int_{\mathbf{R}^{n}} e^{-i z\left(\frac{\xi}{|\xi|} \cdot \eta\right)}|\Phi(\eta)|^{p} d \eta d z .
$$

Since $u(t)=M(t) \mathcal{D}(t) \mathcal{V}(t) \varphi(t)$, to find the large time asymptotics of the solution we need to consider $\mathcal{V}(t) \varphi(t)$. Hence changing the variable of integration $z=\sqrt{t} \eta$ we compute

$$
\begin{aligned}
& \mathcal{V}(t)\left(|\eta|^{-1-\frac{n}{2}(2-p)} A\left(\frac{\eta}{|\eta|}\right)\right) \\
& \quad=t^{\frac{n}{4}\left(2+\frac{2}{n}-p\right)}\left(\frac{i}{2 \pi}\right)^{\frac{n}{2}} \int_{\mathbf{R}^{n}} e^{-\frac{i}{2}|\xi \sqrt{t}-z|^{2}}|z|^{-\frac{n}{2}\left(2+\frac{2}{n}-p\right)} A\left(\frac{z}{|z|}\right) d z .
\end{aligned}
$$

Thus the explicit time growth factor $t^{\frac{n}{4}\left(2+\frac{2}{n}-p\right)}$ appears in the large time asymptotic formula in the subcritical case $p_{s}<p<2+\frac{2}{n}$. We also use the first order differential operator $\mathcal{I}=x \partial_{x}+2 t \partial_{t}$, which helps us to study time decay properties of the Schrödinger evolution group.

The rest of the paper is organized as follows. Section 2 is devoted to the proof of Theorem 1.1. Then in Sect. 3 we prove Theorem 1.2. 


\section{The case of $p>4$}

In the same way as in [9] we multiply both sides of $(1.1)$ by $\mathcal{F} M \mathcal{U}(-t)$ and introduce the new dependent variable

$$
v=e^{-i \frac{\pi}{4}} \mathcal{F} M \mathcal{U}(-t) u=e^{i \frac{\pi}{4}} \bar{E}(t) \mathcal{D}\left(\frac{1}{t}\right) u
$$

to get

$$
\mathcal{L} v=t^{-\frac{p-1}{2}} \bar{E}|v|^{p}, \quad t>1
$$

where $\mathcal{L}=i \partial_{t}+\frac{1}{2 t^{2}} \partial_{\xi}^{2}, E=e^{\frac{i t}{2} \xi^{2}}$. First we state the local existence of solutions to $(2.1)($ see $[2])$.

Theorem 2.1. Let the initial data $v_{0} \in \mathbf{H}^{\nu} \cap \mathbf{H}^{0, \nu}, \frac{1}{2}<\nu<1$. Then for some time $T>1$ there exists a unique solution $v \in \mathbf{C}\left([1, T] ; \mathbf{H}^{\nu} \cap \mathbf{H}^{0, \nu}\right)$ of the Cauchy problem for Eq. (2.1) with initial data $v(1)=v_{0}$.

We denote the fractional derivative for $\alpha \in(0,1)$ (see [14])

$$
\left|\partial_{x}\right|^{\alpha} \phi(x)=\mathcal{F}^{-1}\left(|\xi|^{\alpha} \widehat{\phi}(\xi)\right)=C \int_{\mathbf{R}}(\phi(x-y)-\phi(x)) \frac{d y}{|y|^{1+\alpha}},
$$

where $C=-\frac{\alpha}{2 \Gamma(1-\alpha) \cos \left(\frac{\pi \alpha}{2}\right)}$, and $\Gamma$ is the Euler gamma function.

In the next lemma we find the estimate for $\left\|\left|\partial_{\xi}\right|^{\alpha}\left(\bar{E}|v|^{p}\right)\right\|_{\mathbf{L}^{2}}$.

Lemma 2.2. Let $p>2, \gamma \in(0,1)$. Then we have

$$
\begin{aligned}
\left\|\left|\partial_{\xi}\right|^{\mu}\left(\bar{E}|v|^{p}\right)\right\|_{\mathbf{L}^{2}} \leq & C\langle t\rangle^{\mu+\frac{\gamma}{2}}\left\||\xi|^{\mu+\gamma}|v|^{p}\right\|_{\mathbf{L}^{2}}+C\langle t\rangle^{\frac{\mu}{2}}\|v\|_{\mathbf{L}^{2 p}}^{p} \\
& +C\|v\|_{\mathbf{L}^{\infty}}^{p-1}\left\|\left|\partial_{\xi}\right|^{\mu+\gamma} v\right\|_{\mathbf{L}^{2}}
\end{aligned}
$$

for $\mu \in(0,1)$, and

$$
\begin{aligned}
\left\|\left|\partial_{\xi}\right|^{\nu}\left(\bar{E}|v|^{p}\right)\right\|_{\mathbf{L}^{2}} \leq & C\langle t\rangle^{\nu+\frac{\gamma}{2}}\left\||\xi|^{\nu+\gamma}|v|^{p}\right\|_{\mathbf{L}^{2}}+C\langle t\rangle^{\frac{\nu}{2}}\|v\|_{\mathbf{L}^{2 p}}^{p} \\
& +C\|v\|_{\mathbf{L}^{\infty}}^{p-1}\left\|\left|\partial_{\xi}\right|^{\nu} v\right\|_{\mathbf{L}^{2}}+C\|v\|_{\mathbf{L}^{\infty}}^{p-2}\left\|\left|\partial_{\xi}\right|^{\nu} v\right\|_{\mathbf{L}^{2}}^{2}
\end{aligned}
$$

for $\nu \in\left(\frac{1}{2}, 1\right)$, provided that the right-hand sides are finite.

Proof. By the Leibnitz rule $\left|\partial_{\xi}\right|^{\alpha}(\phi \psi)=\phi\left|\partial_{\xi}\right|^{\alpha} \psi+\left[\left|\partial_{\xi}\right|^{\alpha}, \phi\right] \psi$, where the commutator

$$
\left[\left|\partial_{\xi}\right|^{\alpha}, \phi\right] \psi=C \int_{\mathbf{R}}(\phi(\xi-y)-\phi(\xi)) \psi(\xi-y) \frac{d y}{|y|^{1+\alpha}},
$$

we get

$$
\left|\partial_{\xi}\right|^{\alpha}\left(\bar{E}|v|^{p}\right)=\bar{E}\left|\partial_{\xi}\right|^{\alpha}\left(|v|^{p}\right)+\left[\left|\partial_{\xi}\right|^{\alpha}, \bar{E}\right]|v|^{p} .
$$


We have the estimate for the commutator

$$
\begin{aligned}
& \left\|\left[\left|\partial_{\xi}\right|^{\alpha}, \bar{E}\right]|v|^{p}\right\|_{\mathbf{L}^{2}} \leq C\left\|\int_{|y| \leq\langle t\rangle^{-\frac{1}{2}}}\left(e^{\frac{i t}{2}\left(2(\xi-y) y+y^{2}\right)}-1\right)|v|^{p}(\xi-y) \frac{d y}{|y|^{1+\alpha}}\right\|_{\mathbf{L}^{2}} \\
& +C\left\|\int_{|y| \geq\langle t\rangle^{-\frac{1}{2}}}\left(e^{\frac{i t}{2}\left(2(\xi-y) y+y^{2}\right)}-1\right)|v|^{p}(\xi-y) \frac{d y}{|y|^{1+\alpha}}\right\|_{\mathbf{L}^{2}} \\
& \leq C t^{\alpha+\gamma}\left\|\int_{|y| \leq\langle t\rangle^{-\frac{1}{2}}}|\xi-y|^{\alpha+\gamma}|v|^{p}(\xi-y)|y|^{\gamma-1} d y\right\|_{\mathbf{L}^{2}} \\
& +C t\left\|\int_{|y| \leq\langle t\rangle^{-\frac{1}{2}}}|v|^{p}(\xi-y)|y|^{1-\alpha} d y\right\|_{\mathbf{L}^{2}} \\
& \leq C\langle t\rangle^{\alpha+\frac{\gamma}{2}}\left\||\xi|^{\alpha+\gamma}|v|^{p}\right\|_{\mathbf{L}^{2}}+C\langle t\rangle^{\frac{\alpha}{2}}\|v\|_{\mathbf{L}^{2 p}}^{p} \text {. }
\end{aligned}
$$

Next we consider the estimate for $\left\|\left|\partial_{\xi}\right|^{\alpha}\left(|v|^{p}\right)\right\|_{\mathbf{L}^{2}}$. By the Taylor expansion we have $\left.|| z\right|^{p}-|\zeta|^{p}-\left.p|z|^{p-1}(z-\zeta)|\leq C| z\right|^{p-2}(z-\zeta)^{2}$. Hence we get

$$
\begin{aligned}
\left\||\partial|^{\alpha}|v|^{p}-p|v|^{p-1}|\partial|^{\alpha} v\right\|_{\mathbf{L}^{2}} \leq & C\left\||v(\cdot)|^{p-2} \int_{\mathbf{R}}(v(\cdot-y)-v(\cdot))^{2} \frac{d y}{|y|^{1+\alpha}}\right\|_{\mathbf{L}^{2}} \\
\leq & C\|v\|_{\mathbf{L}^{2 p}}^{p} \int_{|y|>1} \frac{d y}{|y|^{1+\alpha}} \\
& +C \int_{|y|<1}\left\||v(\cdot)|^{p-2}(v(\cdot-y)-v(\cdot))^{2}\right\|_{\mathbf{L}^{2}} \frac{d y}{|y|^{1+\alpha}} .
\end{aligned}
$$

Using the Plancherel theorem we find

$$
\begin{aligned}
\|v(x-y)-v(x)\|_{\mathbf{L}^{2}} & =\left\|\int_{\mathbf{R}^{n}} e^{i x \xi}\left(e^{i y \xi}-1\right) \widehat{v}(\xi) d \xi\right\|_{\mathbf{L}^{2}}=\left\|\left(e^{i y \xi}-1\right) \widehat{v}(\xi)\right\|_{\mathbf{L}^{2}} \\
& \leq C|y|^{\delta}\left\||\xi|^{\delta} \widehat{v}(\xi)\right\|_{\mathbf{L}^{2}}=C|y|^{\delta}\left\|\left|\partial_{\xi}\right|^{\delta} v\right\|_{\mathbf{L}^{2}}
\end{aligned}
$$

for $0 \leq \delta \leq 1$. Then we find the first estimate of the lemma

$$
\begin{aligned}
\left\||\partial|^{\mu}|v|^{p}\right\|_{\mathbf{L}^{2}} \leq & C\left\||v|^{p-1}|\partial|^{\mu} v\right\|_{\mathbf{L}^{2}}+C\|v\|_{\mathbf{L}^{2 p}}^{p} \int_{|y|>1} \frac{d y}{|y|^{1+\mu}} \\
& +C\|v\|_{\mathbf{L}^{\infty}}^{p-1}\left\|\left|\partial_{\xi}\right|^{\mu+\gamma} v\right\|_{\mathbf{L}^{2}} \int_{|y|<1} \frac{d y}{|y|^{1+\mu}} \\
\leq & C\|v\|_{\mathbf{L}^{\infty}}^{p-1}\left\||\partial|^{\mu} v\right\|_{\mathbf{L}^{2}}+C\|v\|_{\mathbf{L}^{2 p}}^{p}+C\|v\|_{\mathbf{L}^{\infty}}^{p-1}\left\|\left|\partial_{\xi}\right|^{\mu+\gamma} v\right\|_{\mathbf{L}^{2}} .
\end{aligned}
$$

By the Sobolev embedding theorem we have $\|v\|_{\mathbf{L}^{4}} \leq C\left\|\left|\partial_{\xi}\right|^{\frac{1}{4}} v\right\|_{\mathbf{L}^{2}}$. Hence

$$
\|v(x-y)-v(x)\|_{\mathbf{L}^{4}}^{2} \leq C\left\|\left|\partial_{\xi}\right|^{\frac{1}{4}}(v(x-y)-v(x))\right\|_{\mathbf{L}^{2}}^{2} \leq|y|^{2 \delta}\left\|\left|\partial_{\xi}\right|^{\frac{1}{4}+\delta} v\right\|_{\mathbf{L}^{2}}^{2}
$$


and

$$
\begin{aligned}
\int_{|y|<1}\|v(x-y)-v(x)\|_{\mathbf{L}^{4}}^{2} \frac{d y}{|y|^{1+\nu}} & \leq C\left\|\left|\partial_{\xi}\right|^{\frac{1}{4}+\delta} v\right\|_{\mathbf{L}^{2}}^{2} \int_{|y|<1}|y|^{2 \delta-1-\nu} d y \\
& \leq C\left\|\left|\partial_{\xi}\right|^{\nu} v\right\|_{\mathbf{L}^{2}}^{2}
\end{aligned}
$$

for $\frac{1}{4}+\delta=\nu<2 \delta$, i.e. $\nu>\frac{1}{2}$. Thus we get the second estimate of the lemma. Lemma 2.2 is proved.

Let us prove a-priori estimates in the norm $\mathbf{H}^{\nu} \cap \mathbf{H}^{0, \nu}$ for the local solutions stated in Theorem 2.1. We assume that $\frac{1}{2}<\nu<1$ and $\gamma>0$ is sufficiently small such that $\frac{1}{2}<\nu \leq \frac{p-1}{2}-1-\frac{3 \gamma}{2}$. Differentiating equation (2.1), we get

$$
\mathcal{L}\left|\partial_{\xi}\right|^{\nu} v=t^{-\frac{p-1}{2}}\left|\partial_{\xi}\right|^{\nu}\left(\bar{E}|v|^{p}\right) .
$$

By Lemma 2.2 we find

$$
\begin{aligned}
\frac{d}{d t}\left\|\left|\partial_{\xi}\right|^{\nu} v\right\|_{\mathbf{L}^{2}} \leq & t^{-\frac{p-1}{2}}\left\|\left|\partial_{\xi}\right|^{\nu}\left(\bar{E}|v|^{p}\right)\right\|_{\mathbf{L}^{2}} \\
\leq & C\langle t\rangle^{\nu-\frac{p-1}{2}+\frac{\gamma}{2}}\left\||\xi|^{\nu+\gamma}|v|^{p}\right\|_{\mathbf{L}^{2}}+C\langle t\rangle^{\frac{\nu}{2}-\frac{p-1}{2}}\|v\|_{\mathbf{L}^{2 \sigma+2}}^{p} \\
& +C t^{-\frac{p-1}{2}}\|v\|_{\mathbf{L}^{\infty}}^{p-1}\left\|\left.\partial_{\xi}\right|^{\nu} v\right\|_{\mathbf{L}^{2}}+C t^{-\frac{p-1}{2}}\|v\|_{\mathbf{L}^{\infty}}^{p-2}\left\|\left.\partial_{\xi}\right|^{\nu} v\right\|_{\mathbf{L}^{2}}^{2} \\
\leq & C\langle t\rangle^{\nu-\frac{p-1}{2}+\frac{\gamma}{2}}\left(\|v\|_{\mathbf{H}^{0, \nu}}+\|v\|_{\mathbf{H}^{\nu}}\right)\|v\|_{\mathbf{H}^{\nu}}^{p-1} .
\end{aligned}
$$

Also we obtain

$$
\begin{aligned}
\mathcal{L}\left(\langle\xi\rangle^{\nu} v\right) & =\langle\xi\rangle^{\nu} \mathcal{L} v+\frac{1}{t^{2}}\left(\partial_{\xi}\langle\xi\rangle^{\nu}\right) \partial_{\xi} v+\frac{1}{2 t^{2}} v \partial_{\xi}^{2}\langle\xi\rangle^{\nu} \\
& =t^{-\frac{p-1}{2}} \bar{E}\langle\xi\rangle^{\nu}|v|^{p}+\frac{1}{t^{2}}\left(\partial_{\xi}\langle\xi\rangle^{\nu}\right) \partial_{\xi} v+\frac{1}{2 t^{2}} v \partial_{\xi}^{2}\langle\xi\rangle^{\nu}
\end{aligned}
$$

Since

$$
\left.\left|\int_{\mathbf{R}}\right| v\right|^{2}\langle\xi\rangle^{\nu} \partial_{\xi}^{2}\langle\xi\rangle^{\nu} d \xi \mid \leq C\|v\|_{\mathbf{L}^{2}}^{2}
$$

and

$$
\begin{aligned}
\left|\int_{\mathbf{R}} \bar{v}\langle\xi\rangle^{\nu}\left(\partial_{\xi}\langle\xi\rangle^{\nu}\right)\left(\partial_{\xi} v\right) d \xi\right| & =\left|\int_{\mathbf{R}} \partial_{\xi}^{1-\nu}\left(\bar{v}\langle\xi\rangle^{\nu}\left(\partial_{\xi}\langle\xi\rangle^{\nu}\right)\right)\left(\partial_{\xi}^{\nu} v\right) d \xi\right| \\
& \leq C\left(\|v\|_{\mathbf{H}^{0, \nu}}+\|v\|_{\mathbf{H}^{\nu}}\right)\|v\|_{\mathbf{H}^{\nu}}
\end{aligned}
$$

we get

$$
\begin{aligned}
\frac{d}{d t}\left(\|v\|_{\mathbf{H}^{0, \nu}}+\left\|\left|\partial_{\xi}\right|^{\nu} v\right\|_{\mathbf{L}^{2}}\right) \leq & C\langle t\rangle^{\nu-\frac{p-1}{2}+\frac{\gamma}{2}}\left(\|v\|_{\mathbf{H}^{0, \nu}}+\|v\|_{\mathbf{H}^{\nu}}\right) \\
& \times\left(\|v\|_{\mathbf{H}^{\nu}}^{p-1}+\|v\|_{\mathbf{H}^{\nu}}\right) .
\end{aligned}
$$

Hence we find the estimate

$$
\|v\|_{\mathbf{H}^{0, \nu}}+\|v\|_{\mathbf{H}^{\nu}} \leq C \varepsilon
$$

since $\nu-\frac{p-1}{2}+\frac{\gamma}{2} \leq-1-\gamma$. In particular we have the estimate

$$
\|u(t)\|_{\mathbf{L}^{q}}=t^{-\frac{1}{2}+\frac{1}{q}}\|v\|_{\mathbf{L}^{q}} \leq C t^{-\frac{1}{2}+\frac{1}{q}}\|v\|_{\mathbf{H}^{\nu}} \leq C \varepsilon t^{-\frac{1}{2}+\frac{1}{q}}
$$


for $2 \leq q \leq \infty$. We next prove the existence of the usual scattering states in $\mathbf{H}^{\nu}$-sense. Denote $\varphi(t)=\mathcal{F U}(-t) u(t)$. We apply the operator $\mathcal{F U}(-t)$ to Eq. (1.1) to get

$$
i \varphi_{t}=\mathcal{F U}(-t)|u|^{p} .
$$

Therefore integrating the last equation with respect to $t$, we obtain

$$
\begin{aligned}
\|\varphi(t)-\varphi(s)\|_{\mathbf{H}^{\nu}} & \leq C \int_{s}^{t}\left\|\mathcal{F U}(-\tau)|u|^{p}\right\|_{\mathbf{H}^{\nu}} d \tau \leq C \int_{s}^{t}\left\|\mathcal{F} M \mathcal{U}(-\tau)|u|^{p}\right\|_{\mathbf{H}^{\nu}} d \tau \\
& \leq C \int_{s}^{t}\left\|\bar{E}|v|^{p}\right\|_{\mathbf{H}^{\nu}} \tau^{-\frac{p-1}{2}} d \tau
\end{aligned}
$$

Then by (2.2) we find

$$
\begin{aligned}
\|\varphi(t)-\varphi(s)\|_{\mathbf{H}^{\nu}} & \leq C \int_{s}^{t}\left\|\bar{E}|v|^{p}\right\|_{\mathbf{H}^{\nu}} \tau^{-\frac{p-1}{2}} d \tau \leq C \varepsilon^{p} \int_{s}^{t} \tau^{\nu-\frac{p-1}{2}+\frac{\gamma}{2}} d \tau \\
& \leq C \varepsilon^{p} s^{-\gamma}
\end{aligned}
$$

for $t>s>1$. Therefore there exists a unique final state $\widehat{u_{+}} \in \mathbf{H}^{\nu}$ such that

$$
\left\|\varphi(t)-\widehat{u_{+}}\right\|_{\mathbf{H}^{\nu}} \leq C \varepsilon^{p} t^{-\gamma}
$$

as $t \rightarrow \infty$. In particular, we have

$$
\begin{aligned}
\left\|u(t)-\mathcal{U}(t) u_{+}\right\|_{\mathbf{L}^{2}} & =\left\|\mathcal{U}(t) \mathcal{F}^{-1} \varphi(t)-\mathcal{U}(t) u_{+}\right\|_{\mathbf{L}^{2}} \\
& =\left\|\varphi(t)-\widehat{u_{+}}\right\|_{\mathbf{L}^{2}} \leq C \varepsilon^{p} t^{-\gamma}
\end{aligned}
$$

as $t \rightarrow \infty$. By the Sobolev inequality

$$
\|\phi\|_{\mathbf{L}^{q}} \leq C\left\|\left|\partial_{x}\right|^{\delta} \phi\right\|_{\mathbf{L}^{2}}^{\frac{1}{\delta}\left(\frac{1}{2}-\frac{1}{q}\right)}\|\phi\|_{\mathbf{L}^{2}}^{1-\frac{1}{\delta}\left(\frac{1}{2}-\frac{1}{q}\right)}
$$

with $\delta>\frac{1}{2}-\frac{1}{q}, 2 \leq q \leq \infty$, we get

$$
\begin{aligned}
& \|(\mathcal{V}(t)-1) \varphi(t)\|_{\mathbf{L}^{q}} \\
& \quad \leq C\left\|\left|\partial_{\xi}\right|^{\delta}(\mathcal{V}(t)-1) \varphi(t)\right\|_{\mathbf{L}^{2}}^{\frac{1}{\delta}\left(\frac{1}{2}-\frac{1}{q}\right)}\|(\mathcal{V}(t)-1) \varphi(t)\|_{\mathbf{L}^{2}}^{1-\frac{1}{\delta}\left(\frac{1}{2}-\frac{1}{q}\right)} \\
& \quad \leq C\left\|\left|\partial_{\xi}\right|^{\delta} \varphi(t)\right\|_{\mathbf{L}^{2}}^{\frac{1}{\delta}\left(\frac{1}{2}-\frac{1}{q}\right)}\|(\mathcal{V}(t)-1) \varphi(t)\|_{\mathbf{L}^{2}}^{1-\frac{1}{\delta}\left(\frac{1}{2}-\frac{1}{q}\right)} .
\end{aligned}
$$

Since $\mathcal{V}(t)=\mathcal{F} M(t) \mathcal{F}^{-1}$ we find

$$
\begin{aligned}
\|(\mathcal{V}(t)-1) \varphi(t)\|_{\mathbf{L}^{2}} & =\left\|\mathcal{F}(M(t)-1) \mathcal{F}^{-1} \varphi(t)\right\|_{\mathbf{L}^{2}} \\
& =\left\|(M(t)-1) \mathcal{F}^{-1} \varphi(t)\right\|_{\mathbf{L}^{2}} \leq C t^{-\frac{\delta}{2}}\left\||x|^{\delta} \mathcal{F}^{-1} \varphi(t)\right\|_{\mathbf{L}^{2}} \\
& \leq C t^{-\frac{\delta}{2}}\left\|\left|\partial_{\xi}\right|^{\delta} \varphi(t)\right\|_{\mathbf{L}^{2}} .
\end{aligned}
$$

Hence we get the estimate

$$
\|(\mathcal{V}(t)-1) \varphi(t)\|_{\mathbf{L}^{q}} \leq C t^{-\frac{1}{2}\left(\delta-\frac{1}{2}+\frac{1}{q}\right)}\left\|\left|\partial_{\xi}\right|^{\delta} \varphi(t)\right\|_{\mathbf{L}^{2}}
$$


with $\delta>\frac{1}{2}-\frac{1}{q}, 2 \leq q \leq \infty$. We now compute the asymptotics of solutions. We have

$$
\begin{aligned}
u(t)= & \mathcal{U}(t) \mathcal{F}^{-1} \varphi(t)=M(t) \mathcal{D}(t) \mathcal{V}(t) \varphi(t)=M(t) \mathcal{D}(t) \widehat{u_{+}} \\
& +M(t) \mathcal{D}(t)\left(\varphi(t)-\widehat{u_{+}}\right)+M(t) \mathcal{D}(t)(\mathcal{V}(t)-1) \varphi(t) .
\end{aligned}
$$

By (2.3) and (2.4) we get

$$
\begin{aligned}
& \left\|u(t)-M(t) \mathcal{D}(t) \widehat{u_{+}}\right\|_{\mathbf{L}^{q}} \\
& \quad \leq t^{-\frac{1}{2}\left(1-\frac{2}{q}\right)}\left\|\varphi(t)-\widehat{u_{+}}\right\|_{\mathbf{L}^{q}}+t^{-\frac{1}{2}\left(1-\frac{2}{q}\right)}\|(\mathcal{V}(t)-1) \varphi(t)\|_{\mathbf{L}^{q}} \\
& \quad \leq C \varepsilon t^{-\frac{1}{2}\left(1-\frac{2}{q}\right)}\left(t^{-\gamma}+t^{-\frac{1}{2}\left(\nu-\frac{1}{2}+\frac{1}{q}\right)}\right)
\end{aligned}
$$

for $2 \leq q \leq \infty$. Theorem 1.1 is proved.

\section{The case of $p_{s}<p<4$}

First let us prove a-priori estimate

$$
\left\|\mathcal{P}^{2} u\right\|_{\mathbf{L}_{t}^{p}\left(\mathbf{I} ; \mathbf{L}_{x}^{p+1}\right)} \leq C \varepsilon
$$

of the local solution from Theorem 2.1 , where $\mathcal{P}^{2}=\left(1, \mathcal{I}, \mathcal{I}^{2}\right)$ and the operator $\mathcal{I}=2 t \partial_{t}+x \partial_{x}$. Denote the space-time norm

$$
\|\phi\|_{\mathbf{L}_{t}^{r}\left(\mathbf{I} ; \mathbf{L}_{x}^{q}\right)}=\|\| \phi(t)\left\|_{\mathbf{L}_{x}^{q}}\right\|_{\mathbf{L}_{t}^{r}(\mathbf{I})},
$$

where $\mathbf{I}$ is a bounded or unbounded time interval. For one dimensional case the Strichartz estimate (see [2], Proposition 2.4.1) can be formulated as follows.

Lemma 3.1. Let $2<r \leq \infty$ and $1<a, b<\infty$ such that $\frac{1}{a}+\frac{1}{b}=\frac{1}{2}-\frac{1}{r}$. Then for any time interval $\mathbf{I}$ the following estimate is true

$$
\left\|\int_{0}^{t} \mathcal{U}(t-\tau) g(\tau) d \tau\right\|_{\mathbf{L}_{t}^{a}\left(\mathbf{I} ; \mathbf{L}_{x}^{r}\right)} \leq C\|g\|_{\mathbf{L}_{t}^{b^{\prime}}\left(\mathbf{I} ; \mathbf{L}_{x}^{r^{\prime}}\right)}
$$

where $b^{\prime}, r^{\prime}$ denote the dual numbers of $b, r$, respectively.

First we use the Strichartz estimate to prove that $u \in \mathbf{L}_{t}^{a}\left([0, T] ; \mathbf{L}_{x}^{p+1}\right)$, where $a>\frac{2(p+1)}{p-1}$. Applying the operator $\mathcal{U}(t-\tau)$ to Eq. (1.1), we get

$$
u(t)=\mathcal{U}(t) u_{0}+\int_{0}^{t} \mathcal{U}(t-\tau)|u(\tau)|^{p} d \tau
$$

Denote

$$
a_{1}=\frac{2(p+1)}{p-1}(1+\varepsilon), \quad a_{2}=\frac{2(p-1)(p+1)}{p+3}>p .
$$

We choose

$$
0<\varepsilon<\frac{p^{2}-3 p-2}{p+3}
$$

so that

$$
a_{1}<a_{2}
$$


We use the Strichartz estimate with $r=p+1, a \in\left[a_{1}, a_{2}\right]$. We let $r^{\prime}=\frac{p+1}{p}$ and

$$
\frac{1}{b^{\prime}}=\frac{p-1}{a_{2}}+\frac{1}{a}
$$

Then

$$
\frac{p}{a_{2}} \leq \frac{1}{b^{\prime}} \leq 1-\frac{p-1}{2(p+1)}\left(\frac{\varepsilon}{1+\varepsilon}\right)
$$

Note that

$$
a_{1} \leq \frac{p}{1-\frac{p-1}{2(p+1)}\left(\frac{\varepsilon}{1+\varepsilon}\right)} \leq p b^{\prime} \leq a_{2}
$$

under the condition (3.2). Thus applying estimate of Lemma 3.1, we get

$$
\begin{aligned}
\left\|\int_{0}^{t} \mathcal{U}(t-\tau)|u(\tau)|^{p} d \tau\right\|_{\mathbf{L}_{t}^{a}\left(\mathbf{I} ; \mathbf{L}_{x}^{p+1}\right)} & \leq\left. C\|\| u\right|^{p}\left\|_{\mathbf{L}_{t}^{b^{\prime}}\left(\mathbf{I} ; \mathbf{L}_{x}^{r^{\prime}}\right)} \leq C\right\| u \|_{\mathbf{L}_{t}^{p b^{\prime}}\left(\mathbf{I} ; \mathbf{L}_{x}^{p+1}\right)}^{p} \\
& \leq C\|u\|_{\mathbf{L}_{t}^{a_{1}}\left(\mathbf{I} ; \mathbf{L}_{x}^{p+1}\right)}+C\|u\|_{\mathbf{L}_{t}^{a_{2}}\left(\mathbf{I} ; \mathbf{L}_{x}^{p+1}\right)}^{p}
\end{aligned}
$$

for all $a \in\left[a_{1}, a_{2}\right]$. Since $\left\|\mathcal{U}(t) u_{0}\right\|_{\mathbf{L}_{x}^{r}} \leq C\langle t\rangle^{\frac{1}{r}-\frac{1}{2}}\left\|u_{0}\right\|_{\mathbf{H}^{1,1}}$ and

$$
\left(\frac{1}{p+1}-\frac{1}{2}\right) a_{1}=-(1+\varepsilon)<-1,
$$

we have

$$
\left\|\mathcal{U}(t) u_{0}\right\|_{\mathbf{L}_{t}^{a}\left(\mathbf{I} ; \mathbf{L}_{x}^{p+1}\right)} \leq C\left\|\langle t\rangle^{\frac{1}{p+1}-\frac{1}{2}}\right\|_{\mathbf{L}_{t}^{a}(\mathbf{I})}\left\|u_{0}\right\|_{\mathbf{H}^{1,1}} \leq C \varepsilon
$$

for all $a \in\left[a_{1}, a_{2}\right]$. Hence the estimate

$$
\sup _{a \in\left[a_{1}, a_{2}\right]}\|u\|_{\mathbf{L}_{t}^{a}\left([0, T] ; \mathbf{L}_{x}^{p+1}\right)} \leq C \varepsilon
$$

follows for all $T>0$. In particular, we find that

$$
\|u\|_{\mathbf{L}_{t}^{p}\left(\mathbf{I} ; \mathbf{L}_{x}^{p+1}\right)} \leq C \varepsilon .
$$

Applying the operator $\mathcal{I}^{2}$ to $(1.1)$, we get $\left(i \partial_{t}+\frac{1}{2} \partial_{x}^{2}\right) \mathcal{I}^{2} u=(\mathcal{I}+2)^{2}|u|^{p}$. Then using the operator $\mathcal{U}(t-\tau)$, and integrating with respect to time, we get

$$
\mathcal{I}^{2} u(t)=\mathcal{U}(t)\left(x \partial_{x}\right)^{2} u_{0}+\int_{0}^{t} \mathcal{U}(t-\tau)(\mathcal{I}+2)^{2}|u|^{p} d \tau
$$

By the Hölder inequality we find

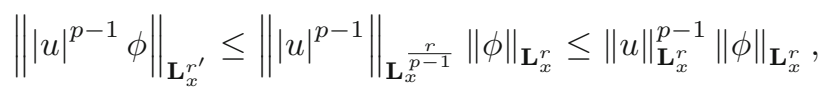

and

$$
\begin{aligned}
\left\||u|^{p-1} \phi\right\|_{\mathbf{L}_{t}^{b^{\prime}}\left(\mathbf{I} ; \mathbf{L}_{x}^{r^{\prime}}\right)} & \leq\|\| u(t)\left\|_{\mathbf{L}_{x}^{r}}^{p-1}\right\| \phi(t)\left\|_{\mathbf{L}_{x}^{r}}\right\|_{\mathbf{L}_{t}^{b^{\prime}}(\mathbf{I})} \\
& \leq\|\| u(t)\left\|_{\mathbf{L}_{x}^{r}}^{p-1}\right\|_{\mathbf{L}_{t}^{\frac{p b^{\prime}}{p-1}}(\mathbf{I})}\|\phi(t)\|_{\mathbf{L}_{x}^{r}}\|\|_{\mathbf{L}_{t}^{p b^{\prime}}(\mathbf{I})}\|\| u\left\|_{\mathbf{L}_{t}^{p-1}\left(\mathbf{I} ; \mathbf{L}_{x}^{p+1}\right)}\right\| \phi \|_{\mathbf{L}_{t}^{p b^{\prime}}\left(\mathbf{I} ; \mathbf{L}_{x}^{p+1}\right)} .
\end{aligned}
$$


As above we use the Strichartz estimate with $r=p+1, a \in\left[a_{1}, a_{2}\right]$ to obtain

$$
\begin{aligned}
& \left\|\int_{0}^{t} \mathcal{U}(t-\tau)(\mathcal{I}+2)|u|^{p} d \tau\right\|_{\mathbf{L}_{t}^{a}\left(\mathbf{I} ; \mathbf{L}_{x}^{p+1}\right)} \leq C\left\|(\mathcal{I}+2)|u|^{p}\right\|_{\mathbf{L}_{t}^{b^{\prime}}\left(\mathbf{I} ; \mathbf{L}_{x}^{p^{\prime}}\right)} \\
& \quad \leq C\|u\|_{\mathbf{L}_{t}^{p b^{\prime}}\left(\mathbf{I} ; \mathbf{L}_{x}^{p+1}\right)}^{p}+C\|u\|_{\mathbf{L}_{t}^{p b^{\prime}}\left(\mathbf{I} ; \mathbf{L}_{x}^{p+1}\right)}^{p}\|\mathcal{I} u\|_{\mathbf{L}_{t}^{p b^{\prime}}\left(\mathbf{I} ; \mathbf{L}_{x}^{p+1}\right)} \leq C \varepsilon .
\end{aligned}
$$

In the same way,

$$
\begin{aligned}
& \left\|\int_{0}^{t} \mathcal{U}(t-\tau)(\mathcal{I}+2)^{2}|u|^{p} d \tau\right\|_{\mathbf{L}_{t}^{a}\left(\mathbf{I} ; \mathbf{L}_{x}^{p+1}\right)} \leq C\left\|(\mathcal{I}+2)^{2}|u|^{p}\right\|_{\mathbf{L}_{t}^{b^{\prime}}\left(\mathbf{I} ; \mathbf{L}_{x}^{p^{\prime}}\right)} \\
& \quad \leq C\|u\|_{\mathbf{L}_{t}^{p b^{\prime}}\left(\mathbf{I} ; \mathbf{L}_{x}^{p+1}\right)}^{p}+C\|u\|_{\mathbf{L}_{t}^{p b^{\prime}}\left(\mathbf{I} ; \mathbf{L}_{x}^{p+1}\right)}^{p-2}\|\|_{\mathbf{L}_{t}^{p b^{\prime}}\left(\mathbf{I} ; \mathbf{L}_{x}^{p+1}\right)}^{2} \\
& \quad+C\|u\|_{\mathbf{L}_{t}^{p b^{\prime}}\left(\mathbf{I} ; \mathbf{L}_{x}^{p+1}\right)}\left\|\mathcal{I}^{2} u\right\|_{\mathbf{L}_{t}^{p b^{\prime}}\left(\mathbf{I} ; \mathbf{L}_{x}^{p+1}\right)} \\
& \quad \leq C \varepsilon .
\end{aligned}
$$

We also have

$$
\left\|\mathcal{U}(t)\left(x \partial_{x}\right)^{2} u_{0}\right\|_{\mathbf{L}_{t}^{a}\left(\mathbf{I} ; \mathbf{L}_{x}^{p+1}\right)} \leq C\left\|\langle t\rangle^{\frac{1}{p+1}-\frac{1}{2}}\right\|_{\mathbf{L}_{t}^{a}(\mathbf{I})}\left\|u_{0}\right\|_{\mathbf{H}^{2,2}} \leq C \varepsilon .
$$

If $u_{0} \in \mathbf{H}^{2,2}$, then the estimate $\sup _{a \in\left[a_{1}, a_{3}\right]}\left\|\mathcal{I}^{2} u\right\|_{\mathbf{L}_{t}^{a}\left([0, T] ; \mathbf{L}_{x}^{p+1}\right)} \leq C \varepsilon$ follows for all $T>0$. Therefore we get the estimate (3.1).

Applying the operator $\mathcal{F U}(-t)$ to (1.1), we have the integral equation

$$
\varphi(t, \xi)=\varphi(1, \xi)-\frac{\sqrt{i}}{\sqrt{2 \pi}} \int_{1}^{t} \tau^{1-\frac{p}{2}} E \int_{\mathbf{R}} e^{-i \tau \xi \eta}|v(\tau, \eta)|^{p} d \eta d \tau,
$$

where $\varphi(t)=\mathcal{F U}(-t) u(t)$ and $v=e^{i \frac{\pi}{4}} \mathcal{F} M \mathcal{U}(-t) u$. We next prove the estimate of the $\mathbf{L}^{l}$-norm of $\varphi$ in terms of the $\mathbf{L}_{t}^{l}\left(\mathbf{I} ; \mathbf{L}_{x}^{q}\right)$-norm of $u$ with $q=\frac{p l}{l-1}$ and

$$
p+1 \leq l<\frac{2}{4-p}
$$

Note that $\frac{2 p}{p-2}<q \leq p+1$ and $p \geq a_{1}=\frac{2(p+1)}{p-1}(1+\varepsilon)$.

Denote $\mathcal{Q}=(1, \widehat{\mathcal{I}}), \mathcal{Q}^{2}=\left(1, \widehat{\mathcal{I}}, \widehat{\mathcal{I}}^{2}\right)$, where $\widehat{\mathcal{I}}=2 t \partial_{t}-\xi \partial_{\xi}$.

Lemma 3.2. The estimate

$$
\left\|\mathcal{Q}^{2} \varphi(t)\right\|_{\mathbf{L}_{t}^{\infty}\left(\mathbf{I} ; \mathbf{L}_{x}^{l}\right)} \leq C\|\varphi(1)\|_{\mathbf{H}^{3,2}}+C\left\|\mathcal{P}^{2} u\right\|_{\mathbf{L}_{t}^{p}\left(\mathbf{I} ; \mathbf{L}_{x}^{q}\right)}^{p}
$$

is true for $\frac{2 p}{p-2}<q \leq p+1$, where $q=\frac{p l}{l-1}$.

Proof. Since $v(t, \xi)=t^{\frac{1}{2}} E u(t, \xi t)$, we have $\|v(t)\|_{\mathbf{L}^{q}}=t^{\frac{1}{2}-\frac{1}{q}}\|u(t)\|_{\mathbf{L}^{q}}$ and

$$
\int_{1}^{t} \tau^{\beta-1-\frac{1}{l}}\|v(\tau)\|_{\mathbf{L}^{q}}^{p} d \tau=\int_{1}^{t}\|u(\tau)\|_{\mathbf{L}^{q}}^{p} d \tau=\|u\|_{\mathbf{L}_{t}^{p}\left(\mathbf{I} ; \mathbf{L}_{x}^{q}\right)}^{p}
$$


with $q=\frac{p l}{l-1}$. We apply the $\mathbf{L}^{l}$-norm to the integral equation and use the estimate $\|\mathcal{F} \phi\|_{\mathbf{L}^{l}} \leq C\|\phi\|_{\mathbf{L}^{\frac{l}{l-1}}}$ for $2 \leq l \leq \infty$, then we get

$$
\begin{aligned}
\left\|\mathcal{Q}^{2} \varphi(t)\right\|_{\mathbf{L}^{l}} \leq & \left\|\left(1,\left(x \partial_{x}\right),\left(x \partial_{x}\right)^{2}\right) \varphi(1)\right\|_{\mathbf{L}^{l}} \\
& +C \int_{1}^{t} \tau^{\beta-1}\left\|\int_{\mathbf{R}} e^{i \tau \xi \eta} \mathcal{Q}^{2}|v(\tau, \eta)|^{p} d \eta\right\|_{\mathbf{L}^{l}} d \tau \\
\leq & C\|\varphi(1)\|_{\mathbf{H}^{3,2}}+C \int_{1}^{t} \tau^{1-\frac{p}{2}-\frac{1}{l}}\left\|\mathcal{F} \mathcal{Q}^{2}|v(\tau)|^{p}\right\|_{\mathbf{L}^{l}} d \tau \\
\leq & C\|\varphi(1)\|_{\mathbf{H}^{3,2}}+C \int_{1}^{t} \tau^{1-\frac{p}{2}-\frac{1}{l}}\left\|\mathcal{Q}^{2}|v(\tau)|^{p}\right\|_{\mathbf{L}^{\frac{l}{l-1}}} d \tau \\
\leq & C\|\varphi(1)\|_{\mathbf{H}^{3,2}}+C \int_{1}^{t} \tau^{1-\frac{p}{2}-\frac{1}{l}}\left\|\mathcal{Q}^{2} v(\tau)\right\|_{\mathbf{L}^{q}}^{p} d \tau \\
\leq & C\|\varphi(1)\|_{\mathbf{H}^{3,2}}+C \|_{\mathcal{P}^{2} u \|_{\mathbf{L}_{t}^{p}\left(\mathbf{I} ; \mathbf{L}_{x}^{q}\right)}^{p}}
\end{aligned}
$$

where $1-\frac{p}{2}-\frac{1}{l}<-1$. Thus we have the estimate of the lemma. Lemma 3.2 is proved.

Now let us prove a-priori estimates of local solutions stated in Theorem 2.1 in the norms

$$
\|v\|_{\mathbf{X}}=\sup _{t \geq 1}\left(t^{-\frac{\beta}{2}}\left\|\langle\xi \sqrt{t}\rangle^{\beta} v(t)\right\|_{\mathbf{L}^{\infty}}+t^{-\frac{\beta}{2}-\gamma}\left\|\langle\xi \sqrt{t}\rangle^{\beta} \mathcal{Q} v(t)\right\|_{\mathbf{L}^{\infty}}\right),
$$

where $\beta=\frac{4-p}{2}, \gamma>0$ is small, and

$$
\|\varphi\|_{\mathbf{Y}}=\sup _{t \geq 1}\left(t^{-\beta}\left\|\langle\xi t\rangle^{\beta} \mathcal{Q} \varphi(t)\right\|_{\mathbf{L}^{\infty}}+t^{1-\beta}\left\|\langle\xi t\rangle^{\beta} \mathcal{Q} \varphi_{t}(t)\right\|_{\mathbf{L}^{\infty}}\right) .
$$

First we show that the estimate of $v(t)$ in the norm $\mathbf{X}$ implies the estimate in the norm $\mathbf{H}^{\mu}$ with $\mu<\frac{p-1}{2}-1$.

Lemma 3.3. Assume that the solution $v$ of (2.1) satisfies the estimate $\|v\|_{\mathbf{X}} \leq$ $C \varepsilon$. Then the following estimate

$$
\|\mathcal{Q} v\|_{\mathbf{L}_{t}^{\infty}\left(\mathbf{I} ; \mathbf{H}^{\mu}\right)} \leq C \varepsilon
$$

is true, where $\mu<\frac{p-3}{2}$.

Proof. By (2.1) we get

$$
\mathcal{L}(\xi v)=t^{-\frac{p-1}{2}} \bar{E} \xi|v|^{p}+t^{-2} v_{\xi}
$$

and

$$
\mathcal{L} v_{\xi}=t^{-\frac{p-1}{2}} \bar{E} \partial_{\xi}|v|^{p}-t^{1-\frac{p-1}{2}} \bar{E} \xi|v|^{p} .
$$

Therefore

and

$$
\frac{d}{d t}\|\xi v\|_{\mathbf{L}^{2}} \leq C t^{-\frac{p-1}{2}}\left\||\xi|^{\beta} v\right\|_{\mathbf{L}^{\infty}}^{p-1}\|\langle\xi\rangle v\|_{\mathbf{L}^{2}}+t^{-2}\left\|v_{\xi}\right\|_{\mathbf{L}^{2}}
$$

$$
\frac{d}{d t}\|v\|_{\mathbf{H}^{1}} \leq C t^{-\frac{p-1}{2}}\|v\|_{\mathbf{L}^{\infty}}^{p-1}\|v\|_{\mathbf{H}^{1}}+C t^{1-\frac{p-1}{2}}\left\||\xi|^{\beta} v\right\|_{\mathbf{L}^{\infty}}^{p-1}\|\langle\xi\rangle v\|_{\mathbf{L}^{2}} .
$$


Hence

$$
\|v\|_{\mathbf{H}^{0,1}}+t^{\frac{p-1}{2}-2}\|v\|_{\mathbf{H}^{1}} \leq C \varepsilon .
$$

By Lemma 2.2 we find

$$
\begin{aligned}
\left\|\left|\partial_{\xi}\right|^{\mu}\left(\bar{E}|v|^{p}\right)\right\|_{\mathbf{L}^{2}} \leq & C\langle t\rangle^{\mu+\frac{\gamma}{2}}\left\||\xi|^{\mu+\gamma}|v|^{p}\right\|_{\mathbf{L}^{2}}+C\langle t\rangle^{\frac{\mu}{2}}\|v\|_{\mathbf{L}^{2 p}}^{p} \\
& +C\|v\|_{\mathbf{L}^{\infty}}^{p-1}\left\|\left|\partial_{\xi}\right|^{\mu+\gamma} v\right\|_{\mathbf{L}^{2}} .
\end{aligned}
$$

By interpolation

$$
\begin{aligned}
\left\|\left|\partial_{\xi}\right|^{\mu+\gamma} v\right\|_{\mathbf{L}^{2}} & \leq C\left\|\left|\partial_{\xi}\right|^{\mu} v\right\|_{\mathbf{L}^{2}}^{1-\frac{\gamma}{1-\mu}}\|v\|_{\mathbf{H}^{1}}^{\frac{\gamma}{1-\mu}} \leq C\left\|\left|\partial_{\xi}\right|^{\mu} v\right\|_{\mathbf{L}^{2}}^{1-\frac{\gamma}{1-\mu}} t^{\left(-\frac{p-1}{2}+2\right) \frac{\gamma}{1-\mu}} \\
& \leq C t^{\gamma}\left\|\left|\partial_{\xi}\right|^{\mu} v\right\|_{\mathbf{L}^{2}}^{1-\frac{\gamma}{1-\mu}} .
\end{aligned}
$$

We have

$$
\begin{aligned}
& \left\||\xi|^{\mu+\gamma}|v|^{p}\right\|_{\mathbf{L}^{2}} \leq\left\||\xi|^{\beta} v\right\|_{\mathbf{L}^{\infty}}^{p-1}\left\||\xi|^{\mu+\gamma-\beta(p-1)} v\right\|_{\mathbf{L}^{2}} \\
& \quad \leq C\left\||\xi|^{\beta} v\right\|_{\mathbf{L}^{\infty}}^{p-1}\|\xi v\|_{\mathbf{L}^{2}}+C\left\||\xi|^{\beta} v\right\|_{\mathbf{L}^{\infty}}^{p}\left\||\xi|^{\mu+\gamma-\beta p}\right\|_{\mathbf{L}^{2}(|\xi|<1)} \leq C \varepsilon^{p} .
\end{aligned}
$$

In the same manner

$$
\|v\|_{\mathbf{L}^{2 p}}^{p} \leq C\|v\|_{\mathbf{L}^{\infty}}^{\frac{p-1}{2}}\|v\|_{\mathbf{L}^{p+1}}^{\frac{p-1}{2}+1} \leq C \varepsilon^{\frac{p-1}{2}} t^{\frac{(p-1) \beta}{4}}\left\|\left|\partial_{\xi}\right|^{\mu} v\right\|_{\mathbf{L}^{2}}^{\frac{p-1}{2}+1} \leq C \varepsilon^{p}\langle t\rangle^{\frac{p-1}{4} \beta} .
$$

Therefore we estimate

$$
\begin{aligned}
\frac{d}{d t}\left\|\left|\partial_{\xi}\right|^{\mu} v\right\|_{\mathbf{L}^{2}} & \leq t^{-\frac{p-1}{2}}\left\|\left|\partial_{\xi}\right|^{\mu}\left(\bar{E}|v|^{p}\right)\right\|_{\mathbf{L}^{2}} \\
& \leq C \varepsilon^{p}\left(\langle t\rangle^{\mu-\frac{p-1}{2}+\gamma}+\langle t\rangle^{\frac{p-1}{2}(\beta-1)+\gamma}+\langle t\rangle^{\frac{\mu}{2}-\frac{p-1}{2}+\frac{p-1}{4} \beta}\right) .
\end{aligned}
$$

Since $\mu<\frac{p-1}{2}-1-2 \gamma, \frac{p-1}{2}(\beta-1)<-1-2 \gamma$, and $\frac{\mu}{2}-\frac{p-1}{2}+\frac{p-1}{4} \beta<-1-2 \gamma$ we get the estimate $\|v\|_{\mathbf{H}^{\mu}} \leq C \varepsilon$. In the same manner, we can consider $\mathcal{Q} v(t)$. Lemma 3.3 is proved.

Next we consider the estimate of $v(t)$ in the norm $\mathbf{X}$.

Lemma 3.4. Let $\|\varphi\|_{\mathbf{Y}} \leq C \varepsilon$ and

$$
\left\|\mathcal{Q}^{2} \varphi\right\|_{\mathbf{L}_{t}^{\infty}\left(\mathbf{I} ; \mathbf{L}_{x}^{l}\right)} \leq C \varepsilon
$$

for $p+1 \leq l<\frac{2}{4-p}$. Then the estimate

$$
\|v\|_{\mathbf{X}} \leq C \varepsilon
$$

is true.

Proof. Note that $v(t)=e^{-i \frac{\pi}{4}} \mathcal{V}(t) \varphi(t)$, where

$$
\mathcal{V}(t) \varphi(t)=\sqrt{\frac{i t}{2 \pi}} \int_{\mathbf{R}} e^{-\frac{i t}{2}(\xi-\eta)^{2}} \varphi(t, \eta) d \eta .
$$


In the domain $|\eta| \leq t^{-\frac{1}{2}}\langle\xi \sqrt{t}\rangle^{-\frac{\beta}{1-\beta}}$ we get

$$
\begin{aligned}
& \sqrt{t} \int_{|\eta| \leq t^{-\frac{1}{2}}\langle\xi \sqrt{t}\rangle^{-\frac{\beta}{1-\beta}}}|\varphi(t, \eta)| d \eta \leq C \sqrt{t}\left\||\xi|^{\beta} \varphi\right\|_{\mathbf{L}^{\infty}} \int_{|\eta| \leq t^{-\frac{1}{2}}\langle\xi \sqrt{t}\rangle^{-\frac{\beta}{1-\beta}}} \frac{d \eta}{|\eta|^{\beta}} \\
& \leq C \varepsilon \sqrt{t} \int_{|\eta| \leq t^{-\frac{1}{2}}\langle\xi \sqrt{t}\rangle^{-\frac{\beta}{1-\beta}}} \frac{d \eta}{|\eta|^{\beta}}=C \varepsilon t^{\frac{\beta}{2}} \int_{|z| \leq\langle\xi \sqrt{t}\rangle^{-\frac{\beta}{1-\beta}}} \frac{d z}{|z|^{\beta}} \leq \frac{C \varepsilon t^{\frac{\beta}{2}}}{\langle\xi \sqrt{t}\rangle^{\beta}} .
\end{aligned}
$$

Using the identity

$$
e^{-\frac{i t}{2}(\xi-\eta)^{2}}=\left(-1+i t(\xi-\eta)^{2}\right)^{-1} \partial_{\eta}\left((\xi-\eta) e^{-\frac{i t}{2}(\xi-\eta)^{2}}\right)
$$

we integrate by parts in the domain $|\eta| \geq t^{-\frac{1}{2}}\langle\xi \sqrt{t}\rangle^{-\frac{\beta}{1-\beta}}$

$$
\begin{aligned}
& \sqrt{\frac{t}{2 \pi}} \int_{|\eta| \geq t^{-\frac{1}{2}}\langle\xi \sqrt{t}\rangle^{-\frac{\beta}{1-\beta}}}\left(-1+i t(\xi-\eta)^{2}\right)^{-1} \partial_{\eta}\left((\xi-\eta) e^{-\frac{i t}{2}(\xi-\eta)^{2}}\right) \varphi(t, \eta) d \eta \\
& =-\sqrt{\frac{t}{2 \pi}} \int_{|\eta| \geq t^{-\frac{1}{2}}\langle\xi \sqrt{t}\rangle^{-\frac{\beta}{1-\beta}}} e^{-\frac{i t}{2}(\xi-\eta)^{2}} \frac{2 i t(\xi-\eta)^{2}}{\left(-1+i t(\xi-\eta)^{2}\right)^{2}} \varphi(t, \eta) d \eta \\
& -\sqrt{\frac{t}{2 \pi}} \int_{|\eta| \geq t^{-\frac{1}{2}}\langle\xi \sqrt{t}\rangle^{-\frac{\beta}{1-\beta}}} e^{-\frac{i t}{2}(\xi-\eta)^{2}} \frac{\xi-\eta}{-1+i t(\xi-\eta)^{2}} \partial_{\eta} \varphi(t, \eta) d \eta \\
& -\left.\sqrt{\frac{t}{2 \pi}} e^{-\frac{i t}{2}(\xi-\eta)^{2}} \frac{\xi-\eta}{-1+i t(\xi-\eta)^{2}} \varphi(t, \eta)\right|_{\eta=-t^{-\frac{1}{2}}\langle\xi \sqrt{t}\rangle^{-\frac{\beta}{1-\beta}}} ^{\eta=t^{-\frac{1}{2}}\langle\xi \sqrt{t}\rangle^{-\frac{\beta}{1-\beta}}}
\end{aligned}
$$

$\operatorname{Via}|\varphi(t, \xi)| \leq C \varepsilon t^{\beta}\langle\xi t\rangle^{-\beta}$, we estimate

$$
\begin{aligned}
& \left|\sqrt{\frac{t}{2 \pi}} e^{-\frac{i t}{2}(\xi-\eta)^{2}} \frac{\xi-\eta}{-1+i t(\xi-\eta)^{2}} \varphi(t, \eta)\right|_{\eta=-t^{-\frac{1}{2}}\langle\xi \sqrt{t}\rangle^{-\frac{\beta}{1-\beta}}}^{\eta=t^{-\frac{1}{2}}\langle\xi \sqrt{t}\rangle^{-\frac{\beta}{1-\beta}}} \mid \\
& \quad \leq C \varepsilon t^{\beta}\langle\xi t\rangle^{-\beta} \leq \frac{C \varepsilon t^{\frac{\beta}{2}}}{\langle\xi \sqrt{t}\rangle^{\beta}}
\end{aligned}
$$

and

$$
\begin{aligned}
& \left|\sqrt{\frac{t}{2 \pi}} \int_{|\eta| \geq t^{-\frac{1}{2}}\langle\xi \sqrt{t}\rangle^{-\frac{\beta}{1-\beta}}} e^{-\frac{i t}{2}(\xi-\eta)^{2}} \frac{2 i t(\xi-\eta)^{2}}{\left(-1+i t(\xi-\eta)^{2}\right)^{2}} \varphi(t, \eta) d \eta\right| \\
& \quad \leq C \varepsilon t^{\frac{1}{2}} t^{\beta} \int_{|\eta| \geq t^{-\frac{1}{2}}\langle\xi \sqrt{t}\rangle^{-\frac{\beta}{1-\beta}}} \frac{d \eta}{\left(1+(\xi \sqrt{t}-\eta \sqrt{t})^{2}\right)\langle\eta t\rangle^{\beta}} \\
& \leq C \varepsilon t^{\beta} \int_{|z| \geq\langle\xi \sqrt{t}\rangle^{-\frac{\beta}{1-\beta}}} \frac{d z}{\langle\xi \sqrt{t}-z\rangle^{2}\langle z \sqrt{t}\rangle^{\beta}} \\
& \quad \leq C \varepsilon t^{\frac{\beta}{2}} \int_{|z| \geq\langle\xi \sqrt{t}\rangle^{-\frac{\beta}{1-\beta}}} \frac{d z}{\langle\xi \sqrt{t}-z\rangle^{2}|z|^{\beta}} \leq \frac{C \varepsilon t^{\frac{\beta}{2}}}{\langle\xi \sqrt{t}\rangle^{\beta}} .
\end{aligned}
$$


Then changing $\partial_{\eta} \varphi=\frac{1}{\eta}\left(\widehat{\mathcal{I}} \varphi+2 t \partial_{t} \varphi\right)$ we get

$$
\begin{aligned}
& \left|\sqrt{\frac{i t}{2 \pi}} \int_{|\eta| \geq t^{-\frac{1}{2}}\langle\xi \sqrt{t}\rangle^{-\frac{\beta}{1-\beta}}} e^{-\frac{i t}{2}(\xi-\eta)^{2}} \frac{\xi-\eta}{-1+i t(\xi-\eta)^{2}} \partial_{\eta} \varphi(t, \eta) d \eta\right| \\
& \leq C \int_{|\eta| \geq t^{-\frac{1}{2}}\langle\xi \sqrt{t}\rangle^{-\frac{\beta}{1-\beta}}} \frac{1}{|\eta|\langle\xi \sqrt{t}-\eta \sqrt{t}\rangle}|\mathcal{Q} \varphi(t, \eta)| d \eta \\
& \quad+C \int_{|\eta| \geq t^{-\frac{1}{2}}\langle\xi \sqrt{t}\rangle^{-\frac{\beta}{1-\beta}}} \frac{1}{|\eta|\langle\xi \sqrt{t}-\eta \sqrt{t}\rangle}\left|t \varphi_{t}(t, \eta)\right| d \eta \\
& \leq C \varepsilon t^{\frac{\beta}{2}} \int_{|z| \geq\langle\xi \sqrt{t}\rangle^{-\frac{\beta}{1-\beta}}} \frac{\left.d z\right|^{1+\beta}\langle\xi \sqrt{t}-z\rangle}{\langle\xi \sqrt{t}\rangle^{\frac{\beta}{2}}} .
\end{aligned}
$$

Thus the estimate $\sup _{t \geq 1} t^{-\frac{\beta}{2}}\left\|\langle\xi \sqrt{t}\rangle^{\beta} v(t)\right\|_{\mathbf{L}^{\infty}} \leq C \varepsilon$ follows. In order to estimate $\mathcal{Q} v$ we write

$$
\mathcal{Q} v=e^{-i \frac{\pi}{4}} \mathcal{V}(t) \mathcal{Q} \varphi(t)=\sqrt{\frac{t}{2 \pi}} \int_{\mathbf{R}} e^{-\frac{i t}{2}(\xi-\eta)^{2}} \mathcal{Q} \varphi(t, \eta) d \eta
$$

Then as above

$$
\begin{aligned}
& \sqrt{t} \int_{|\eta| \leq t^{-\frac{1}{2}}\langle\xi \sqrt{t}\rangle^{-\frac{\beta}{1-\beta}}}|\mathcal{Q} \varphi(t, \eta)| d \eta \\
& \quad \leq C \sqrt{t}\left\||\xi|^{\beta} \mathcal{Q} \varphi\right\|_{\mathbf{L}^{\infty}} \int_{|\eta| \leq t^{-\frac{1}{2}}\langle\xi \sqrt{t}\rangle^{-\frac{\beta}{1-\beta}}} \frac{d \eta}{|\eta|^{\beta}} \leq \frac{C \varepsilon t^{\frac{\beta}{2}}}{\langle\xi \sqrt{t}\rangle^{\beta}}
\end{aligned}
$$

and

$$
\begin{aligned}
& \sqrt{\frac{t}{2 \pi}} \int_{|\eta| \geq t^{-\frac{1}{2}}}\langle\xi \sqrt{t}\rangle^{-\frac{\beta}{1-\beta}}\left(-1+i t(\xi-\eta)^{2}\right)^{-1} \partial_{\eta}\left((\xi-\eta) e^{-\frac{i t}{2}(\xi-\eta)^{2}}\right) \mathcal{Q} \varphi(t, \eta) d \eta \\
& =-\sqrt{\frac{t}{2 \pi}} \int_{|\eta| \geq t^{-\frac{1}{2}}\langle\xi \sqrt{t}\rangle^{-\frac{\beta}{1-\beta}}} e^{-\frac{i t}{2}(\xi-\eta)^{2}} \frac{2 i t(\xi-\eta)^{2}}{\left(-1+i t(\xi-\eta)^{2}\right)^{2}} \mathcal{Q} \varphi(t, \eta) d \eta \\
& \quad-\sqrt{\frac{t}{2 \pi}} \int_{|\eta| \geq t^{-\frac{1}{2}}\langle\xi \sqrt{t}\rangle^{-\frac{\beta}{1-\beta}}} e^{-\frac{i t}{2}(\xi-\eta)^{2}} \frac{\xi-\eta}{-1+i t(\xi-\eta)^{2}} \partial_{\eta} \mathcal{Q} \varphi(t, \eta) d \eta \\
& -\left.\sqrt{\frac{t}{2 \pi}} e^{-\frac{i t}{2}(\xi-\eta)^{2}} \frac{\xi-\eta}{-1+i t(\xi-\eta)^{2}} \mathcal{Q} \varphi(t, \eta)\right|_{\eta=-t^{-\frac{1}{2}}\langle\xi \sqrt{t}\rangle^{-\frac{\beta}{1-\beta}}\langle\xi \sqrt{t}\rangle^{-\frac{\beta}{1-\beta}}} ^{\eta=}
\end{aligned}
$$

$B y|\mathcal{Q} \varphi(t, \xi)| \leq C \varepsilon t^{\beta}\langle\xi t\rangle^{-\beta}$, we estimate

$$
\left|\sqrt{\frac{t}{2 \pi}} e^{-\frac{i t}{2}(\xi-\eta)^{2}} \frac{\xi-\eta}{-1+i t(\xi-\eta)^{2}} \mathcal{Q} \varphi(t, \eta)\right|_{\eta=-t^{-\frac{1}{2}}\langle\xi \sqrt{t}\rangle^{-\frac{\beta}{1-\beta}}}^{\eta=t^{-\frac{1}{2}}\langle\xi \sqrt{t}\rangle^{-\frac{\beta}{1-\beta}}} \mid \leq \frac{C \varepsilon t^{\frac{\beta}{2}}}{\langle\xi \sqrt{t}\rangle^{\beta}}
$$


and

$\left|\sqrt{\frac{t}{2 \pi}} \int_{|\eta| \geq t^{-\frac{1}{2}}\langle\xi \sqrt{t}\rangle^{-\frac{\beta}{1-\beta}}} e^{-\frac{i t}{2}(\xi-\eta)^{2}} \frac{2 i t(\xi-\eta)^{2}}{\left(-1+i t(\xi-\eta)^{2}\right)^{2}} \mathcal{Q} \varphi(t, \eta) d \eta\right| \leq \frac{C \varepsilon t^{\frac{\beta}{2}}}{\langle\xi \sqrt{t}\rangle^{\beta}}$.

Then changing $\partial_{\eta} \mathcal{Q} \varphi=\frac{1}{\eta}\left(\widehat{\mathcal{I}} \mathcal{Q} \varphi+2 t \partial_{t} \mathcal{Q} \varphi\right)$ we get

$$
\begin{aligned}
& \left|\sqrt{\frac{t}{2 \pi}} \int_{|\eta| \geq t^{-\frac{1}{2}}\langle\xi \sqrt{t}\rangle^{-\frac{\beta}{1-\beta}}} e^{-\frac{i t}{2}(\xi-\eta)^{2}} \frac{\xi-\eta}{-1+i t(\xi-\eta)^{2}} \partial_{\eta} \mathcal{Q} \varphi(t, \eta) d \eta\right| \\
& \leq C \int_{|\eta| \geq t^{-\frac{1}{2}}\langle\xi \sqrt{t}\rangle^{-\frac{\beta}{1-\beta}}} \frac{1}{|\eta|\langle\xi \sqrt{t}-\eta \sqrt{t}\rangle}\left|\mathcal{Q}^{2} \varphi(t, \eta)\right| d \eta \\
& \quad+C \int_{|\eta| \geq t^{-\frac{1}{2}}\langle\xi \sqrt{t}\rangle^{-\frac{\beta}{1-\beta}}} \frac{1}{|\eta|\langle\xi \sqrt{t}-\eta \sqrt{t}\rangle}\left|t \mathcal{Q} \varphi_{t}(t, \eta)\right| d \eta .
\end{aligned}
$$

We have

$$
\begin{aligned}
& \int_{|\eta| \geq t^{-\frac{1}{2}}\langle\xi \sqrt{t}\rangle^{-\frac{\beta}{1-\beta}}} \frac{1}{|\eta|\langle\xi \sqrt{t}-\eta \sqrt{t}\rangle}\left|t \mathcal{Q} \varphi_{t}(t, \eta)\right| d \eta \\
& \quad \leq C \varepsilon t^{\frac{\beta}{2}} \int_{|z| \geq\langle\xi \sqrt{t}\rangle^{-\frac{\beta}{1-\beta}}} \frac{d z}{|z|^{1+\beta}\langle\xi \sqrt{t}-z\rangle} \leq C \varepsilon t^{\frac{\beta}{2}}\langle\xi \sqrt{t}\rangle^{-1+\frac{\beta^{2}}{1-\beta}} \leq \frac{C \varepsilon t^{\frac{\beta}{2}}}{\langle\xi \sqrt{t}\rangle^{\beta}}
\end{aligned}
$$

since $\frac{-1+\beta+\beta^{2}}{1-\beta}<-\beta$ for $\beta=\frac{4-p}{2}<\frac{1}{2}$. Finally applying the estimate $\| \mathcal{Q}^{2}$ $\varphi(t) \|_{\mathbf{L}^{l}} \leq C \varepsilon$ and using the Hölder inequality, we obtain with $q=\frac{l}{l-1}$ and $\frac{1}{l}=\beta+\frac{\gamma}{2}$

$$
\begin{aligned}
& \int_{|\eta| \geq t^{-\frac{1}{2}}\langle\xi \sqrt{t}\rangle^{-\frac{\beta}{1-\beta}}} \frac{1}{|\eta|\langle\xi \sqrt{t}-\eta \sqrt{t}\rangle}\left|\mathcal{Q}^{2} \varphi(t, \eta)\right| d \eta \\
& \leq\left\|\mathcal{Q}^{2} \varphi(t)\right\|_{\mathbf{L}^{l}}\left(\int_{|\eta| \geq t^{-\frac{1}{2}}\langle\xi \sqrt{t}\rangle^{-\frac{\beta}{1-\beta}}} \frac{\left.d \eta\right|^{q}\langle\xi \sqrt{t}-\eta \sqrt{t}\rangle^{q}}{\mid \frac{1}{q}}\right. \\
& \leq t^{\frac{1}{2 l}}\left\|\mathcal{Q}^{2} \varphi(t)\right\|_{\mathbf{L}^{l}}\left(\int_{|z| \geq\langle\xi \sqrt{t}\rangle^{-\frac{\beta}{1-\beta}}} \frac{d z}{|z|^{q}\langle\xi \sqrt{t}-z\rangle^{q}}\right)^{\frac{1}{q}} \\
& \leq C\langle\xi \sqrt{t}\rangle^{-1+\frac{\beta}{1-\beta} \frac{1}{l}} t^{\frac{1}{2 l}}\left\|\mathcal{Q}^{2} \varphi(t)\right\|_{\mathbf{L}^{l}} \\
& \leq C\langle\xi \sqrt{t}\rangle^{\frac{-1+\beta+\beta^{2}}{1-\beta}+\frac{\beta}{1-\beta} \frac{\gamma}{2}} t^{\frac{\beta}{2}+\gamma}\left\|\mathcal{Q}^{2} \varphi(t)\right\|_{\mathbf{L}^{l}} \leq \frac{C \varepsilon t^{\frac{\beta}{2}+\gamma}}{\langle\xi \sqrt{t}\rangle^{\beta}}
\end{aligned}
$$

since $\frac{-1+\beta+\beta^{2}}{1-\beta}<-\beta$. Lemma 3.4 is proved.

Finally we estimate $\varphi$ in the norm $\mathbf{Y}$.

Lemma 3.5. Suppose that $\|v\|_{\mathbf{X}} \leq C \varepsilon$ and

$$
\|\mathcal{Q} v\|_{\mathbf{L}_{t}^{\infty}\left(\mathbf{I} ; \mathbf{H}^{\mu}\right)} \leq C \varepsilon
$$


where $\frac{-p^{2}+4 p+1}{2(p+1)}<\mu<\frac{p-3}{2}$. Then the estimate

$$
\|\varphi\|_{\mathbf{Y}}<C \varepsilon
$$

is true.

Proof. By the integral equation

$$
\varphi(t, \xi)=\varphi(1, \xi)-\frac{1}{\sqrt{2 \pi}} \int_{1}^{t} \tau^{\beta-1} E \int_{\mathbf{R}} e^{-i \tau \xi \eta}|v(\tau, \eta)|^{p} d \eta d \tau
$$

we have

$$
\left\|\langle t \xi\rangle^{\beta} \mathcal{Q} \varphi(t)\right\|_{\mathbf{L}^{\infty}} \leq\left\|\langle t \xi\rangle^{\beta} \varphi(1)\right\|_{\mathbf{L}^{\infty}}+C\left\|\langle t \xi\rangle^{\beta} \int_{1}^{t} \tau^{\beta-1} \mathcal{K} d \tau\right\|_{\mathbf{L}^{\infty}},
$$

where $\beta=\frac{4-p}{2}$ and

$$
\mathcal{K}=E \int_{\mathbf{R}} e^{-i \tau \xi \eta} \mathcal{Q}|v(\tau, \eta)|^{p} d \eta
$$

By the Sobolev inequality $\|v\|_{\mathbf{L}^{r}} \leq C\|v\|_{\mathbf{H}^{\mu}} \leq C \varepsilon$ with $\frac{1}{r} \geq \frac{1}{2}-\mu$, i.e. $2 \leq r \leq$ $p+1$, we have

$$
\begin{aligned}
\left|\int_{1}^{t} \tau^{\beta-1} \mathcal{K} d \tau\right| & \leq\left.\left|\int_{1}^{t} \tau^{\beta-1} E \int_{\mathbf{R}} e^{-i \tau \xi \eta} \mathcal{Q}\right| v(\tau, \eta)\right|^{p} d \eta d \tau \mid \\
& \leq C \int_{1}^{t} \tau^{\beta-1}\|\mathcal{Q} v(\tau)\|_{\mathbf{L}^{p}}^{p} d \tau \leq C \sup _{\tau \geq 0}\|\mathcal{Q} v(\tau)\|_{\mathbf{L}^{p}}^{p} \int_{1}^{t} \tau^{\beta-1} d \tau \\
& \leq C t^{\beta} \sup _{\tau \geq 0}\|\mathcal{Q} v(\tau)\|_{\mathbf{L}^{p}}^{p} .
\end{aligned}
$$

We choose $\lambda>\beta$ such that $\lambda+\frac{p-3}{2(p+1)} \leq \mu<\frac{p-1}{2}-1$. By the definition $|\xi|^{\lambda} \mathcal{F} \phi=\mathcal{F}\left|\partial_{\xi}\right|^{\lambda} \phi$

$$
\begin{aligned}
\left\|\left(1+|\tau \xi|^{\lambda}\right) \mathcal{K}\right\|_{\mathbf{L}^{\infty}} & =\left\|\int_{\mathbf{R}} e^{-i \tau \xi \eta}\left(1+\left|\partial_{\eta}\right|^{\lambda}\right) \mathcal{Q}|v(\tau, \eta)|^{p} d \eta\right\|_{\mathbf{L}^{\infty}} \\
& \leq\left\|\left(1+\left|\partial_{\eta}\right|^{\lambda}\right) \mathcal{Q}|v(\tau, \eta)|^{p}\right\|_{\mathbf{L}^{1}} \\
& \leq\|\mathcal{Q} v\|_{\mathbf{L}^{p}}^{p}+\tau^{\beta-1}\left\|\left|\partial_{\xi}\right|^{\lambda} \mathcal{Q}|v|^{p}\right\|_{\mathbf{L}^{1}} \leq C \varepsilon^{p},
\end{aligned}
$$

since by the Hölder and Sobolev inequalities

$$
\left\|\left|\partial_{\xi}\right|^{\lambda} \mathcal{Q}|v|^{p}\right\|_{\mathbf{L}^{1}} \leq C\|\mathcal{Q} v\|_{\mathbf{H}^{\mu}}^{p} \leq C \varepsilon^{p} .
$$

Then

$$
\begin{aligned}
& t^{-\beta}\left\|\langle t \xi\rangle^{\beta} \mathcal{Q} \varphi(t)\right\|_{\mathbf{L}^{\infty}} \leq t^{-\beta}\left\|\langle t \xi\rangle^{\beta} \varphi(1)\right\|_{\mathbf{L}^{\infty}}+C t^{-\beta}\left\|\langle t \xi\rangle^{\beta} \int_{1}^{t} \mathcal{K} d \tau\right\|_{\mathbf{L}^{\infty}} \\
& \leq C \varepsilon+C t^{-\beta}\left\|\langle t \xi\rangle^{\beta} \int_{1}^{t} \tau^{\beta-1}\left(1+|\tau \xi|^{\lambda}\right)^{-1} d \tau\right\|_{\mathbf{L}^{\infty} \tau \geq 1} \sup _{\tau \geq 1}\left\|\left(1+|\tau \xi|^{\lambda}\right) \mathcal{K}\right\|_{\mathbf{L}^{\infty}} \\
& \leq C \varepsilon+C \varepsilon^{p}\left\|\int_{|\xi|}^{|\xi| t} \frac{z^{\beta-1} d z}{(1+z)^{\lambda}}\right\|_{\mathbf{L}^{\infty}} \leq C \varepsilon+C \varepsilon^{p} .
\end{aligned}
$$


In the same manner we get

$$
t^{1-\beta}\left\|\langle\xi t\rangle^{\lambda} \mathcal{Q} \varphi_{t}(t)\right\|_{\mathbf{L}^{\infty}} \leq C \varepsilon
$$

Lemma 3.5 is proved.

Using the estimate (3.1), Lemmas $3.3-3.5$ and the standard continuation argument, we obtain a global solution $u \in \mathbf{C}\left(\mathbf{R}^{+}, \mathbf{H}^{3,2}\right)$ to the Cauchy problem (1.1) which satisfies the estimate

$$
\|v\|_{\mathbf{X}}+\|\varphi\|_{\mathbf{Y}}+\|\mathcal{Q} v\|_{\mathbf{L}_{t}^{\infty}\left(\mathbf{I} ; \mathbf{H}^{\mu}\right)}+\left\|\mathcal{Q}^{2} \varphi\right\|_{\mathbf{L}_{t}^{\infty}\left(\mathbf{I} ; \mathbf{L}_{x}^{l}\right)}+\left\|\mathcal{P}^{2} u\right\|_{\mathbf{L}_{t}^{p}\left(\mathbf{I} ; \mathbf{L}_{x}^{p+1}\right)}<C \varepsilon
$$

where $p+1 \leq l<\frac{2}{4-p}, \frac{-p^{2}+4 p+1}{2(p+1)}<\mu<\frac{p-3}{2}$. In particular, since $v(t, \xi)=$ $t^{\frac{1}{2}} E u(t, \xi t)$, by the Sobolev inequality we have

$$
\|u(t)\|_{\mathbf{L}^{l}} \leq C t^{\frac{1}{l}-\frac{1}{2}}\|v(t)\|_{\mathbf{L}^{l}} \leq C t^{\frac{1}{l}-\frac{1}{2}}\|v(t)\|_{\mathbf{H}^{\mu}} \leq C \varepsilon t^{\frac{1}{l}-\frac{1}{2}}
$$

for $2 \leq l<\frac{2}{4-p}$, and

$$
\begin{aligned}
\|u(t)\|_{\mathbf{L}^{l}} & \leq t^{\frac{1}{l}-\frac{1}{2}}\|v(t)\|_{\mathbf{L}^{l}} \\
& \leq t^{\frac{1}{l}-\frac{1}{2}}\left\|\langle\xi \sqrt{t}\rangle^{\frac{4-p}{2}} v(t)\right\|_{\mathbf{L}^{\infty}}\left\|\langle\xi \sqrt{t}\rangle^{-\frac{4-p}{2}}\right\|_{\mathbf{L}^{l}} \leq C \varepsilon t^{\frac{1}{2 l}-\frac{1}{2}+\frac{4-p}{4}}
\end{aligned}
$$

for $\frac{2}{4-p}<l \leq \infty$.

Let us now prove the existence of the usual scattering states. We apply the operator $\mathcal{F U}(-t)$ to $(1.1)$ to get

$$
i \varphi_{t}=\mathcal{F U}(-t)|u|^{p}
$$

for all $t>0$. Therefore integrating (3.3) with respect to $t$, we obtain as in the proof of Lemma 3.3

$$
\begin{aligned}
& \|\varphi(t)-\varphi(s)\|_{\mathbf{H}^{\mu}} \leq C \int_{s}^{t}\left\|\mathcal{F U}(-\tau)|u|^{p}\right\|_{\mathbf{H}^{\mu}} d \tau \\
& \leq C \int_{s}^{t}\left\|\mathcal{V}(\tau) \mathcal{F U}(-\tau)|u|^{p}\right\|_{\mathbf{H}^{\mu}} d \tau \leq C \int_{s}^{t}\left\|\mathcal{F} M \mathcal{U}(-\tau)|u|^{p}\right\|_{\mathbf{H}^{\mu}} d \tau \\
& \quad \leq C \int_{s}^{t}\left\|\bar{E}|v|^{p}\right\|_{\mathbf{H}^{\mu}} \tau^{-\frac{\sigma}{2}} d \tau \leq C \int_{s}^{t} \tau^{-1-\gamma} d \tau \leq C s^{-\gamma}
\end{aligned}
$$

for $t>s>0$. Hence there exists a unique final state $\widehat{u_{+}} \in \mathbf{H}^{\mu}$ such that

$$
\left\|\varphi(t)-\widehat{u_{+}}\right\|_{\mathbf{H}^{\mu}} \leq C \varepsilon t^{-\gamma}
$$

as $t \rightarrow \infty$. Thus by (3.4), the Sobolev inequality and (2.4) with $2 \leq q<\frac{1}{\frac{1}{2}-\mu}$ we get

$$
\begin{aligned}
& \left\|u(t)-M(t) \mathcal{D}(t) \widehat{u_{+}}\right\|_{\mathbf{L}^{q}} \\
& \quad \leq t^{-\frac{1}{2}\left(1-\frac{2}{q}\right)}\left\|\varphi(t)-\widehat{u_{+}}\right\|_{\mathbf{L}^{q}}+t^{-\frac{1}{2}\left(1-\frac{2}{q}\right)}\|(\mathcal{V}(t)-1) \varphi(t)\|_{\mathbf{L}^{q}} \\
& \quad \leq C \varepsilon t^{-\frac{1}{2}\left(1-\frac{2}{q}\right)}\left(t^{-\gamma}+t^{-\frac{1}{2}\left(\mu-\frac{1}{2}+\frac{1}{q}\right)}\right)
\end{aligned}
$$

for $2 \leq q \leq \frac{2}{4-p}-\gamma$ with some $\gamma>0$. 
Finally we need to prove the asymptotics of solutions in $\mathbf{L}^{q}$ with $q>\frac{2}{4-p}$. By the integral equation for $\varphi$ we get

$$
\begin{aligned}
\varphi(t, \xi) & =\varphi(1, \xi)-\frac{1}{\sqrt{2 \pi}} \int_{1}^{t} \tau^{\beta-1} E \int_{\mathbf{R}} e^{-i \tau \xi \eta}|v(\tau, \eta)|^{p} d \eta d \tau \\
& =\Phi(\xi)+R(t, \xi)
\end{aligned}
$$

where

$$
\begin{aligned}
\Phi(\xi) & =\varphi(1, \xi)-\frac{1}{\sqrt{2 \pi}} \int_{1}^{\infty} \tau^{\beta-1} E \int_{\mathbf{R}} e^{-i \tau \xi \eta}|v(\tau, \eta)|^{p} d \eta d \tau, \\
R(t, \xi) & =\frac{1}{\sqrt{2 \pi}} \int_{t}^{\infty} \tau^{\beta-1} E \int_{\mathbf{R}} e^{-i \tau \xi \eta}|v(\tau, \eta)|^{p} d \eta d \tau,
\end{aligned}
$$

and $\beta=\frac{4-p}{2}$. We can estimate

$$
|\Phi(\xi)| \leq C \varepsilon+C \varepsilon^{p}|\xi|^{-\beta}
$$

and

$$
\begin{aligned}
|R(t, \xi)| & \leq\left. C\left|\int_{t}^{\infty} \tau^{\beta-1}\langle\tau \xi\rangle^{-\beta-\gamma} \int_{\mathbf{R}} e^{-i \tau \xi \eta}\left\langle\partial_{\eta}\right\rangle^{\beta+\gamma}\right| v(\tau, \eta)\right|^{p} d \eta d \tau \mid \\
& \leq C \varepsilon^{p}|\xi|^{-\beta}\left|\int_{t}^{\infty} \frac{(\tau \xi)^{\beta-1}}{\langle\tau \xi\rangle^{\beta+\gamma}} d \xi \tau\right| \\
& \leq C \varepsilon^{p}|\xi|^{-\beta}\langle t \xi\rangle^{-\gamma} \leq C \varepsilon^{p} t^{-\gamma}|\xi|^{-\beta-\gamma} .
\end{aligned}
$$

Let us estimate the operator $\mathcal{V}(t)$, where

$$
\mathcal{V}(t) \varphi(t)=\sqrt{\frac{i t}{2 \pi}} \int_{\mathbf{R}} e^{-\frac{i t}{2}(\xi-\eta)^{2}} \varphi(t, \eta) d \eta .
$$

We have

$$
\begin{aligned}
& \left\|i^{\frac{1}{2}} v(t)-\varphi(t)\right\|_{\mathbf{H}^{\mu-2 \gamma}}=\|(\mathcal{V}(t)-1) \varphi(t)\|_{\mathbf{H}^{\mu-2 \gamma}} \\
& \quad=\left\|\mathcal{F}(M(t)-1) \mathcal{F}^{-1} \varphi(t)\right\|_{\mathbf{H}^{\mu-2 \gamma}} \\
& \quad=\left\|(M(t)-1) \mathcal{F}^{-1} \varphi(t)\right\|_{\mathbf{H}^{0, \mu-2 \gamma}} \leq C t^{-\frac{\mu}{2}}\left\||x|^{\mu} \mathcal{F}^{-1} \varphi(t)\right\|_{\mathbf{H}^{0, \mu-2 \gamma}} \\
& \quad \leq C t^{-\frac{\lambda}{2}}\left\|\mathcal{F}^{-1} \varphi(t)\right\|_{\mathbf{H}^{0, \mu}}=C t^{-\gamma}\|\varphi(t)\|_{\mathbf{H}^{\mu}} .
\end{aligned}
$$

Hence

$$
\Phi(\xi)=\varphi(1, \xi)-\frac{1}{\sqrt{2 \pi}} \int_{1}^{\infty} \tau^{\beta-1} E \int_{\mathbf{R}} e^{-i \tau \xi \eta}|\varphi(\tau, \eta)|^{p} d \eta d \tau+R_{1} .
$$

As in the proof of Lemma 3.5 we estimate the remainder term $R_{1}$ as

$$
\begin{aligned}
\left|R_{1}(t, \xi)\right| & =\left|\frac{1}{\sqrt{2 \pi}} \int_{1}^{\infty} \tau^{\beta-1} E \int_{\mathbf{R}} e^{-i \tau \xi \eta}\left(|\varphi(\tau, \eta)|^{p}-|v(\tau, \eta)|^{p}\right) d \eta d \tau\right| \\
& \leq C \int_{1}^{\infty} \tau^{\beta-1}(1+\tau|\xi|)^{-\lambda}\|v(\tau)-\varphi(\tau)\|_{\mathbf{H}^{\mu-2 \gamma}}\|v(\tau)\|_{\mathbf{H}^{\mu}}^{p-1} d \tau \\
& \leq C|\xi|^{\gamma-\beta} \int_{1}^{\infty} \tau^{-1}(1+\tau|\xi|)^{-\lambda}(\tau|\xi|)^{\beta-\gamma}\|\varphi(t)\|_{\mathbf{H}^{\mu}}\|v(\tau)\|_{\mathbf{H}^{\mu}}^{p-1} d \tau \\
& \leq C \varepsilon^{p}|\xi|^{\gamma-\beta},
\end{aligned}
$$


where $\lambda>\beta$ is such that $\lambda+\frac{p-3}{2(p+1)} \leq \mu<\frac{p-1}{2}-1$. Then we find

$$
\begin{aligned}
& \frac{1}{\sqrt{2 \pi}} \int_{1}^{\infty} \tau^{\beta-1} E \int_{\mathbf{R}} e^{-i \tau \xi \eta}|\varphi(\tau, \eta)|^{p} d \eta d \tau \\
& =\frac{1}{\sqrt{2 \pi}} \int_{1}^{\infty} \tau^{\beta-1} \int_{\mathbf{R}} e^{-i \tau \xi \eta}|\Phi(\eta)|^{p} d \eta d \tau+R_{2}+R_{3}
\end{aligned}
$$

As in the proof of Lemma 3.5 we get the estimates of remainder terms as

$$
\begin{aligned}
\left|R_{3}\right| & =\left.\left|\frac{1}{\sqrt{2 \pi}} \int_{1}^{\infty} \tau^{\beta-1}(E-1) \int_{\mathbf{R}} e^{-i \tau \xi \eta}\right| \varphi(\tau, \eta)\right|^{p} d \eta d \tau \mid \\
& \leq C \int_{1}^{\infty} \tau^{\beta-1}|E-1|(1+\tau|\xi|)^{-\lambda}\|\varphi(\tau, \eta)\|_{\mathbf{H}^{\mu}}^{p} d \tau \\
& \leq C|\xi|^{\gamma-\beta} \int_{1}^{\infty} \tau^{-1}(\tau|\xi|)^{\beta}(\tau|\xi|)^{\gamma}(1+\tau|\xi|)^{-\lambda} d \tau \leq C \varepsilon^{p}|\xi|^{\gamma-\beta}
\end{aligned}
$$

and

$$
\begin{aligned}
\left|R_{2}\right| & =\left|\frac{1}{\sqrt{2 \pi}} \int_{1}^{\infty} \tau^{\beta-1} \int_{\mathbf{R}} e^{-i \tau \xi \eta}\left(|\varphi(\tau, \eta)|^{p}-|\Phi(\eta)|^{p}\right) d \eta d \tau\right| \\
& \leq C \int_{1}^{\infty} \tau^{\beta-1}(1+\tau|\xi|)^{-\lambda}\|\varphi(\tau)-\Phi\|_{\mathbf{H}^{\mu}}\left(\|\varphi(\tau)\|_{\mathbf{H}^{\lambda}}^{p-1}+\|\Phi\|_{\mathbf{H}^{\lambda}}^{p-1}\right) d \tau \\
& \leq C \int_{1}^{\infty} \tau^{\beta-1-\gamma}(1+\tau|\xi|)^{-\lambda} d \tau \leq C \varepsilon^{p}|\xi|^{\gamma-\beta} .
\end{aligned}
$$

Changing $\tau|\xi|=z$, we find the main term

$$
\begin{aligned}
& \frac{1}{\sqrt{2 \pi}} \int_{0}^{\infty} \tau^{\beta-1} \int_{\mathbf{R}} e^{-i \tau \xi \eta}|\Phi(\eta)|^{p} d \eta d \tau \\
& =\frac{1}{\sqrt{2 \pi}}|\xi|^{-\beta} \int_{0}^{\infty} z^{\beta-1} \int_{\mathbf{R}} e^{-i \frac{\xi}{|\xi|} z \eta}|\Phi(\eta)|^{p} d \eta d z \\
& =\frac{1}{\sqrt{2 \pi}}|\xi|^{-\beta} A\left(\frac{\xi}{|\xi|}\right),
\end{aligned}
$$

where

$$
\begin{aligned}
A(1) & =\int_{0}^{\infty} z^{\beta-1} \int_{\mathbf{R}} e^{-i z \eta}|\Phi(\eta)|^{p} d \eta d z \\
& =\int_{\mathbf{R}}|\Phi(\eta)|^{p} d \eta \int_{0}^{\infty} e^{-i z \eta} z^{\beta-1} d z \\
A(-1) & =\int_{0}^{\infty} z^{\beta-1} \int_{\mathbf{R}} e^{i z \eta}|\Phi(\eta)|^{p} d \eta d z=\overline{A(1)} .
\end{aligned}
$$

By [1] it is known that

$$
\int_{0}^{\infty} e^{-i z \eta} z^{\beta-1} d z=|\eta|^{-\beta} \Gamma(\beta)\left(\cos \left(\frac{\pi \beta}{2}\right)-\frac{i \eta}{|\eta|} \sin \left(\frac{\pi \beta}{2}\right)\right) .
$$

Hence

$$
A(1)=\int_{\mathbf{R}}|\Phi(\eta)|^{p}|\eta|^{-\beta} \Gamma(\beta)\left(\cos \left(\frac{\pi \beta}{2}\right)-\frac{i \eta}{|\eta|} \sin \left(\frac{\pi \beta}{2}\right)\right) d \eta
$$


and $A(-1)=\overline{A(1)}$. Note that $A(1)=0$ only if $\Phi(\eta)=0$ for all $\eta \in \mathbf{R}$, which can not happen.

Thus we prove the asymptotics

$$
\varphi(t, \xi)=|\xi|^{-\beta} A\left(\frac{\xi}{|\xi|}\right)+O\left(\varepsilon|\xi|^{\gamma-\beta}\right) .
$$

In the same manner we get

$$
\mathcal{I} \varphi(t, \xi)=|\xi|^{-\beta} A_{1}\left(\frac{\xi}{|\xi|}\right)+O\left(\varepsilon|\xi|^{\gamma-\beta}\right) .
$$

Then as in the proof of Lemma 3.4 we use the identity

$$
e^{-\frac{i t}{2}(\xi-\eta)^{2}}=\left(-1+i t(\xi-\eta)^{2}\right)^{-1} \partial_{\eta}\left((\xi-\eta) e^{-\frac{i t}{2}(\xi-\eta)^{2}}\right)
$$

and integration by parts to find that

$$
\begin{aligned}
i^{\frac{1}{2}} v(t, \xi) & =\mathcal{V}(t) \varphi(t) \\
= & \sqrt{\frac{i t}{2 \pi}} \int_{\mathbf{R}}\left(-1+i t(\xi-\eta)^{2}\right)^{-1} \partial_{\eta}\left((\xi-\eta) e^{\left.-\frac{i t}{2}(\xi-\eta)^{2}\right) \varphi(t, \eta) d \eta}\right. \\
= & \sqrt{\frac{i t}{2 \pi}} \int_{|\eta| \leq t^{-\frac{1}{2}}\langle\xi \sqrt{t}\rangle^{-\frac{\beta}{1-\beta}}} e^{-\frac{i t}{2}(\xi-\eta)^{2}} \varphi(t, \eta) d \eta \\
& -\left.\sqrt{\frac{i t}{2 \pi}} \frac{\xi-\eta}{-1+i t(\xi-\eta)^{2}} e^{-\frac{i t}{2}(\xi-\eta)^{2}} \varphi(t, \eta)\right|_{\eta=-t^{-\frac{1}{2}}\langle\xi \sqrt{t}\rangle^{-\frac{\beta}{1-\beta}}} ^{\eta=t^{-\frac{1}{2}}\langle\xi \sqrt{t}\rangle^{-\frac{\beta}{1-\beta}}} \\
& -\sqrt{\frac{i t}{2 \pi}} \int_{|\eta| \geq t^{-\frac{1}{2}}\langle\xi \sqrt{t}\rangle^{-\frac{\beta}{1-\beta}}} e^{-\frac{i t}{2}(\xi-\eta)^{2}} \frac{2 i t(\xi-\eta)^{2}}{\left(-1+i t(\xi-\eta)^{2}\right)^{2}} \varphi(t, \eta) d \eta \\
& -\sqrt{\frac{i t}{2 \pi}} \int_{|\eta| \geq t^{-\frac{1}{2}}\langle\xi \sqrt{t}\rangle^{-\frac{\beta}{1-\beta}}} e^{-\frac{i t}{2}(\xi-\eta)^{2}} \frac{\xi-\eta}{\eta\left(-1+i t(\xi-\eta)^{2}\right)} \widehat{\mathcal{I}} \varphi(t, \eta) d \eta \\
& -\sqrt{\frac{i t}{2 \pi}} \int_{|\eta| \geq t^{-\frac{1}{2}}\langle\xi \sqrt{t}\rangle^{-\frac{\beta}{1-\beta}}} e^{-\frac{i t}{2}(\xi-\eta)^{2}} \frac{\xi-\eta}{\eta\left(-1+i t(\xi-\eta)^{2}\right)} t \partial_{t} \varphi(t, \eta) d \eta .
\end{aligned}
$$

We have

$$
\begin{aligned}
& \sqrt{\frac{i t}{2 \pi}} \int_{|\eta| \leq t^{-\frac{1}{2}}\langle\xi \sqrt{t}\rangle^{-\frac{\beta}{1-\beta}}} e^{-\frac{i t}{2}(\xi-\eta)^{2}} \varphi(t, \eta) d \eta \\
& =\sqrt{\frac{i t}{2 \pi}} \int_{|\eta| \leq t^{-\frac{1}{2}}\langle\xi \sqrt{t}\rangle^{-\frac{\beta}{1-\beta}}} e^{-\frac{i t}{2}(\xi-\eta)^{2}}|\eta|^{-\beta} A\left(\frac{\eta}{|\eta|}\right) d \eta \\
& \quad+\sqrt{\frac{i t}{2 \pi}} \int_{|\eta| \leq t^{-\frac{1}{2}}\langle\xi \sqrt{t}\rangle^{-\frac{\beta}{1-\beta}}} e^{-\frac{i t}{2}(\xi-\eta)^{2}} O\left(\varepsilon|\eta|^{-\beta+\gamma}\right) d \eta
\end{aligned}
$$


Vol. 21 (2014)

$$
\begin{aligned}
= & t^{\frac{\beta}{2}} \sqrt{\frac{i}{2 \pi}} \int_{|z| \leq\langle\xi \sqrt{t}\rangle^{-\frac{\beta}{1-\beta}}} e^{-\frac{i}{2}(\xi \sqrt{t}-z)^{2}}|z|^{-\beta} A\left(\frac{z}{|z|}\right) d z \\
& +O\left(\varepsilon t^{\frac{\beta}{2}-\frac{\gamma}{2}}\langle\xi \sqrt{t}\rangle^{-\beta}\right) .
\end{aligned}
$$

In the same manner, we obtain

$$
\begin{aligned}
& \left.\sqrt{\frac{i t}{2 \pi}} \frac{\xi-\eta}{-1+i t(\xi-\eta)^{2}} e^{-\frac{i t}{2}(\xi-\eta)^{2}} \varphi(t, \eta)\right|_{\eta=-t^{-\frac{1}{2}}\langle\xi \sqrt{t}\rangle^{-\frac{\beta}{1-\beta}}} ^{\eta=t^{-\frac{1}{2}}\langle\xi \sqrt{t}\rangle^{-\frac{\beta}{1-\beta}}} \\
& =\left.t^{\frac{\beta}{2}} \sqrt{\frac{i}{2 \pi}} \frac{(\xi \sqrt{t}-z) e^{-\frac{i}{2}(\xi \sqrt{t}-z)^{2}}}{-1+i(\xi \sqrt{t}-z)^{2}}|z|^{-\beta} A\left(\frac{z}{|z|}\right)\right|_{z=-\langle\xi \sqrt{t}\rangle^{-\frac{\beta}{1-\beta}}} ^{z=\langle\xi \sqrt{t}\rangle^{-\frac{\beta}{1-\beta}}} \\
& +O\left(\varepsilon t^{\frac{\beta}{2}-\frac{\gamma}{2}}\langle\xi \sqrt{t}\rangle^{-\beta}\right)
\end{aligned}
$$

and

$$
\begin{aligned}
& \sqrt{\frac{i t}{2 \pi}} \int_{|\eta| \geq t^{-\frac{1}{2}}\langle\xi \sqrt{t}\rangle^{-\frac{\beta}{1-\beta}}} e^{-\frac{i t}{2}(\xi-\eta)^{2}} \frac{2 i t(\xi-\eta)^{2}}{\left(-1+i t(\xi-\eta)^{2}\right)^{2}} \varphi(t, \eta) d \eta \\
& =\sqrt{\frac{i t}{2 \pi}} \int_{|\eta| \geq t^{-\frac{1}{2}}\langle\xi \sqrt{t}\rangle^{-\frac{\beta}{1-\beta}}} e^{-\frac{i t}{2}(\xi-\eta)^{2}} \frac{2 i t(\xi-\eta)^{2}}{\left(-1+i t(\xi-\eta)^{2}\right)^{2}}|\eta|^{-\beta} A\left(\frac{\eta}{|\eta|}\right) d \eta \\
& \quad+\sqrt{\frac{i t}{2 \pi}} \int_{|\eta| \geq t^{-\frac{1}{2}}\langle\xi \sqrt{t}\rangle^{-\frac{\beta}{1-\beta}}} e^{-\frac{i t}{2}(\xi-\eta)^{2}} \frac{2 i t(\xi-\eta)^{2}}{\left(-1+i t(\xi-\eta)^{2}\right)^{2}} O\left(\varepsilon^{p}|\eta|^{-\beta+\gamma}\right) d \eta \\
& =t^{\frac{\beta}{2}} \Psi_{1}(\xi \sqrt{t})+O\left(\varepsilon t^{\frac{\beta}{2}-\frac{\gamma}{2}}\langle\xi \sqrt{t}\rangle^{-\beta}\right)
\end{aligned}
$$

where

$$
\begin{aligned}
& \Psi_{1}(\xi \sqrt{t}) \\
& =t^{-\frac{\beta}{2}} \sqrt{\frac{i t}{2 \pi}} \int_{|\eta| \geq t^{-\frac{1}{2}}\langle\xi \sqrt{t}\rangle^{-\frac{\beta}{1-\beta}}} e^{-\frac{i t}{2}(\xi-\eta)^{2}} \frac{2 i t(\xi-\eta)^{2}}{\left(-1+i t(\xi-\eta)^{2}\right)^{2}}|\eta|^{-\beta} A\left(\frac{\eta}{|\eta|}\right) d \eta \\
& \quad=\sqrt{\frac{i}{2 \pi}} \int_{|z| \geq\langle\xi \sqrt{t}\rangle^{-\frac{\beta}{1-\beta}}} e^{-\frac{i}{2}(\xi \sqrt{t}-z)^{2}} \frac{2 i(\xi \sqrt{t}-z)^{2}}{|z|^{\beta}\left(-1+i(\xi \sqrt{t}-z)^{2}\right)^{2}} A\left(\frac{z}{|z|}\right) d z .
\end{aligned}
$$


Then changing $\partial_{\eta} \varphi=\frac{1}{\eta}\left(\widehat{\mathcal{I}} \varphi+2 t \partial_{t} \varphi\right)$ we get

$$
\begin{aligned}
\sqrt{\frac{i t}{2 \pi}} & \int_{|\eta| \geq t^{-\frac{1}{2}}\langle\xi \sqrt{t}\rangle^{-\frac{\beta}{1-\beta}}} e^{-\frac{i t}{2}(\xi-\eta)^{2}} \frac{\xi-\eta}{\eta\left(-1+i t(\xi-\eta)^{2}\right)} \widehat{\mathcal{I}} \varphi(t, \eta) d \eta \\
= & \sqrt{\frac{i t}{2 \pi}} \int_{|\eta| \geq t^{-\frac{1}{2}}\langle\xi \sqrt{t}\rangle^{-\frac{\beta}{1-\beta}}} e^{-\frac{i t}{2}(\xi-\eta)^{2}} \frac{\xi-\eta}{\eta\left(-1+i t(\xi-\eta)^{2}\right)}|\eta|^{-\beta} A_{1}\left(\frac{\eta}{|\eta|}\right) d \eta \\
& +\sqrt{\frac{i t}{2 \pi}} \int_{|\eta| \geq t^{-\frac{1}{2}}\langle\xi \sqrt{t}\rangle^{-\frac{\beta}{1-\beta}}} e^{-\frac{i t}{2}(\xi-\eta)^{2}} \frac{\xi-\eta}{\eta\left(-1+i t(\xi-\eta)^{2}\right)} O\left(\varepsilon^{p}|\eta|^{-\beta+\gamma}\right) d \eta \\
= & t^{\frac{\beta}{2}} \Psi_{2}(\xi \sqrt{t})+O\left(\varepsilon t^{\frac{\beta}{2}-\frac{\gamma}{2}}\langle\xi \sqrt{t}\rangle^{-\beta}\right)
\end{aligned}
$$

where

$$
\begin{aligned}
& \Psi_{2}(\xi \sqrt{t}) \\
& =t^{-\frac{\beta}{2}} \sqrt{\frac{i t}{2 \pi}} \int_{|\eta| \geq t^{-\frac{1}{2}}\langle\xi \sqrt{t}\rangle^{-\frac{\beta}{1-\beta}}} e^{-\frac{i t}{2}(\xi-\eta)^{2}} \frac{\xi-\eta}{\eta\left(-1+i t(\xi-\eta)^{2}\right)}|\eta|^{-\beta} A_{1}\left(\frac{\eta}{|\eta|}\right) d \eta \\
& =\sqrt{\frac{i}{2 \pi}} \int_{|z| \geq\langle\xi \sqrt{t}\rangle^{-\frac{\beta}{1-\beta}}} e^{-\frac{i}{2}(\xi \sqrt{t}-z)^{2}} \frac{\xi \sqrt{t}-z}{z|z|^{\beta}\left(-1+i(\xi \sqrt{t}-z)^{2}\right)} A_{1}\left(\frac{z}{|z|}\right) d z .
\end{aligned}
$$

We also have the estimate

$$
\begin{aligned}
& \sqrt{\frac{i t}{2 \pi}} \int_{|\eta| \geq t^{-\frac{1}{2}}\langle\xi \sqrt{t}\rangle^{-\frac{\beta}{1-\beta}}} e^{-\frac{i t}{2}(\xi-\eta)^{2}} \frac{\xi-\eta}{\eta\left(-1+i t(\xi-\eta)^{2}\right)} t \partial_{t} \varphi(t, \eta) d \eta \\
& =\sqrt{\frac{i t}{2 \pi}} \int_{|\eta| \geq t^{-\frac{1}{2}}\langle\xi \sqrt{t}\rangle^{-\frac{\beta}{1-\beta}}} e^{-\frac{i t}{2}(\xi-\eta)^{2}} \frac{\xi-\eta}{\eta\left(-1+i t(\xi-\eta)^{2}\right)} O\left(\varepsilon t^{-\gamma}|\eta|^{-\beta-\gamma}\right) d \eta \\
& =O\left(\varepsilon t^{\frac{\beta}{2}-\frac{\gamma}{2}}\langle\xi \sqrt{t}\rangle^{-\beta}\right) .
\end{aligned}
$$

We apply (3.6)-(3.10) to (3.5) to get the asymptotics for the solution

$$
\begin{aligned}
& \left\|u(t)-e^{\frac{i x^{2}}{2 t}} \frac{1}{\sqrt{i t}} t^{1-\frac{p}{4}} \Psi_{+}\left(\frac{x}{\sqrt{t}}\right)\right\|_{\mathbf{L}^{q}} \\
& =\left\|u(t)-t^{1-\frac{p}{4}} M(t) \mathcal{D}(t) \Psi(\xi \sqrt{t})\right\|_{\mathbf{L}^{q}} \\
& \leq C \varepsilon t^{\frac{1}{2}-\frac{p}{4}-\gamma}\left\|\left\langle\frac{x}{\sqrt{t}}\right\rangle^{-\beta}\right\|_{\mathbf{L}^{q}} \leq \varepsilon t^{\frac{1}{2}-\frac{p}{4}+\frac{1}{2 q}-\gamma}
\end{aligned}
$$

for $\frac{2}{4-p}<q \leq \infty$, where 


$$
\begin{aligned}
\Psi(\xi \sqrt{t})= & \sqrt{\frac{i}{2 \pi}} \int_{|z|<\langle\xi \sqrt{t}\rangle^{-\frac{\beta}{1-\beta}}} e^{-\frac{i}{2}(\xi \sqrt{t}-z)^{2}}|z|^{-\beta} A\left(\frac{z}{|z|}\right) d z \\
& -\left.t^{\frac{\beta}{2}} \sqrt{\frac{i}{2 \pi}} \frac{(\xi \sqrt{t}-z) e^{-\frac{i}{2}(\xi \sqrt{t}-z)^{2}}}{-1+i(\xi \sqrt{t}-z)^{2}}|z|^{-\beta} A\left(\frac{z}{|z|}\right)\right|_{z=-\langle\xi \sqrt{t}\rangle^{-\frac{\beta}{1-\beta}}} ^{z=\langle\xi \sqrt{t}\rangle^{-\frac{\beta}{1-\beta}}} \\
& +\Psi_{1}(\xi \sqrt{t})+\Psi_{2}(\xi \sqrt{t})
\end{aligned}
$$

since $u(t)=M(t) \mathcal{D}(t) i^{\frac{1}{2}} v(t)$. Theorem 1.2 is proved.

\section{Acknowledgments}

The work of N.H. is supported by JSPS KAKENHI Grant Numbers 24654034, 25220702. The work of P.I.N. is partially supported by CONACYT and PAPIIT project IN100113. We are grateful to an unknown referee for many useful suggestions and comments.

\section{References}

[1] Bateman, H., Erdelyi, A.: Tables of Integral Transforms. McGraw-Hill Book Co., NY (1954)

[2] Cazenave, Th.: Semilinear Schrödinger equations. Courant Institute of Mathematical Sciences. American Mathematical Society, New York, Providence, RI (2003)

[3] Glassey, R.T.: Existence in the large for $\square u=F(u)$ in two space dimensions. Math. Z. 178, 233-261 (1981)

[4] Glassey, R.T.: Finite-time blow up for solutions of nonlinear wave equations. Math. Z. 177, 323-340 (1981)

[5] Georgiev, V., Lindblad, H., Sogge, C.D.: Weighted Strichartz estimates and global existence for semilinear wave equations. Am. J. Math 119, 1291-1319 (1997)

[6] John, F.: Blow-up of solutions of nonlinear wave equations in three space dimensions. Manuscr. Math. 28, 235-268 (1979)

[7] Hayashi, N., Naumkin, P.I.: Asymptotics for large time of solutions to nonlinear Schrödinger and Hartree equations. Am. J. Math. 120, 369-389 (1998)

[8] Hayashi, N., Naumkin, P.I.: Remark on the global existence and large time asymptotics of solutions for the quadratic NLS. Nonlinear Anal. 74(18), 69506964 (2011)

[9] Hayashi, N., Ozawa, T.: Scattering theory in the weighted $\mathbf{L}^{2}\left(\mathbf{R}^{n}\right)$ spaces for some Schrödinger equations. Ann. I.H.P. (Phys. Théor.) 48, 17-37 (1998) 
[10] Ikeda, M., Wakasugi, Y.: Small data blow-up of $\mathbf{L}^{2}$-solution for the nonlinear Schrödinger equation without gauge invariance. Differ. Integral Equ. 26, 12751285 (2013)

[11] Ozawa, T.: Long range scattering for nonlinear Schrödinger equations in one space dimension. Commun. Math. Phys. 139, 479-493 (1991)

[12] Schaeffer, J.: The equation $\square u=|u|^{p}$ for the critical value of $p$. Proc. R. Soc. Edinb. 101, 31-44 (1985)

[13] Sideris, T.: Nonexistence of global solutions to semilinear wave equations in high dimensions. J. Differ. Equ. 52(3), 378-406 (1984)

[14] Stein, E.M.: Singular Integrals and Differentiability Properties of Functions, vol. 30. Princeton Univ. Press, Princeton (1970)

[15] Strauss, W.A.: Nonlinear Scattering Theory, Scattering Theory in Mathematical Physics. Reidel, Dordrect, pp. 53-79 (1979)

[16] Strauss, W.A.: Nonlinear scattering theory at low energy. J. Funct. Anal. 41, 110-133 (1981)

[17] Strauss, W.A.: Nonlinear Wave Equations. CBMS Regional Conference Series in Math., vol. 73. AMS (1989)

[18] Yordanov, B., Zhang, Q.S.: Finite time blow up for critical wave equations in high dimensions. J. Funct. Anal. 231, 361-374 (2006)

[19] Zhang, Q.S.: Blow-up results for nonlinear parabolic equations on manifolds. Duke Math. J. 97, 515-539 (1999)

N. Hayashi

Department of Mathematics

Graduate School of Science

Osaka University

Osaka Toyonaka 560-0043

Japan

e-mail:nhayashi@math.sci.osaka-u.ac.jp

Pavel I. Naumkin

Centro de Ciencias Matemáticas

UNAM Campus Morelia

AP 61-3 (Xangari)

Morelia CP 58089

Michoacán

Mexico

e-mail: pavelni@matmor.unam.mx

Received: 7 November 2012.

Accepted: 21 October 2013. 